\title{
Identifying and characterising health policy and system-relevant documents in Uganda: a scoping review to develop a framework for the development of a one-stop shop
}

Boniface Mutatina ${ }^{1 *}$, Robert Basaza ${ }^{1,2}$, Ekwaro Obuku $^{1,5}$, John N. Lavis ${ }^{3,4+}$ and Nelson Sewankambo ${ }^{1+}$

\begin{abstract}
Background: Health policymakers in low- and middle-income countries continue to face difficulties in accessing and using research evidence for decision-making. This study aimed to identify and provide a refined categorisation of the policy documents necessary for building the content of a one-stop shop for documents relevant to health policy and systems in Uganda. The on-line resource is to facilitate timely access to well-packaged evidence for decision-making.

Methods: We conducted a scoping review of Uganda-specific, health policy, and systems-relevant documents produced between 2000 and 2014. Our methods borrowed heavily from the 2005 Arksey and O'Malley approach for scoping reviews and involved five steps, which that include identification of the research question; identification of relevant documents; screening and selection of the documents; charting of the data; and collating, summarising and reporting results. We searched for the documents from websites of relevant government institutions, non-governmental organisations, health professional councils and associations, religious medical bureaus and research networks. We presented the review findings as numerical analyses of the volume and nature of documents and trends over time in the form of tables and charts.
\end{abstract}

Results: We identified a total of 265 documents including policies, strategies, plans, guidelines, rapid response summaries, evidence briefs for policy, and dialogue reports. The top three clusters of national priority areas addressed in the documents were governance, coordination, monitoring and evaluation (28\%); disease prevention, mitigation, and control (23\%); and health education, promotion, environmental health and nutrition (15\%). The least addressed were curative, palliative care, rehabilitative services and health infrastructure, each addressed in three documents (1\%), and early childhood development in one document. The volume of documents increased over the past 15 years; however, the distribution of the different document types over time has not been uniform.

Conclusion: The review findings are necessary for mobilising and packaging the local policy-relevant documents in Uganda in a one-stop shop; where policymakers could easily access them to address pressing questions about the health system and interventions. The different types of available documents and the national priority areas covered provide a good basis for building and organising the content in a meaningful way for the resource.

Keywords: Evidence-informed policy, Health policy and system documents, Framework, Clearinghouse, Uganda, Lowand middle-income countries

\footnotetext{
* Correspondence: bonifacemutatina@yahoo.com

${ }^{\dagger}$ Equal contributors

${ }^{1}$ College of Health Sciences, Makerere University, Kampala, Uganda

Full list of author information is available at the end of the article
} 


\section{Background}

Linking health research evidence to action is one of the many important components of national and global responses to contemporary public health challenges $[1,2]$. It is important for both making evidence-informed policies $^{1}$ and decisions ${ }^{2}$ on health services and improving the health systems within which the interventions and services are provided [3]. When policies and decisions are made without considering the best available evidence, it may waste resources and opportunities, and possibly do more harm than good [4]. In the recent past, there has been a strong emphasis worldwide on reflecting the best available evidence in health policies and decisions $[2,5]$. However, more evidence may not necessarily mean better policies [6, 7]. Greenhalgh et al. [7] notes that a better policy is what is appropriate in the circumstances in agreement with the overall desirable goal. Research evidence is one of the necessary inputs into policymaking, which may also be influenced by context and other factors [8].

Linking research to action in low- and middle-income countries has remained a challenge, despite the international attention and significant efforts to address it $[3,9]$. Health policymakers ${ }^{3}$ and stakeholders ${ }^{4}$ continue to face difficulties in accessing and using research evidence for policy and decision-making [10]. They are often unable to rapidly identify decision-relevant information when pressing issues emerge, partly due to the lack of one-stop shops with optimally packaged evidence [11]. By making evidence available, a one-stop shop becomes one of the necessary inputs for increasing access to evidence. Although it may not be a sufficient factor, its absence creates a clear gap and thus a barrier to use of evidence by policy and decisionmakers [11]. The one-stop shop may facilitate timely access to well-packaged evidence by policy and decision-makers when faced with questions about health systems and interventions [12]. In a systematic review on health policymakers' perceptions of their use of evidence, Innvaer et al. [10] identified timely access to evidence as one of the facilitators of use of evidence in policymaking. This is corroborated by Lavis et al. [11] and further supported by Oliver et al. [13] in an updated systematic review. The latter included perceptions of other stakeholder groups such as researchers, managers and research users other than policymakers [13].

In the recent past, there have been efforts to develop one-stop shops for both global research evidence and local policy-relevant documents to address questions about health interventions and health systems in high-income countries. Examples include The Cochrane Library, Health Systems Evidence, NHS Library and Knowledge Center and HTA Database Canada Search Interface [14-17]. On the other hand, resources focused on local policy-relevant documents are lacking in low- and middle-income countries and the feasibility of developing them has not been tested.
To address this challenge in Uganda, in 2011, the Supporting the Use of Research Evidence (SURE) in African health systems project [18] embarked on developing the Uganda Clearinghouse for Health Policy and Systems - a one-stop shop for health policy-relevant documents. The SURE project was a collaborative project that built on and supported Evidence-Informed Policy Networks (EVIPNets) in Africa and the Regional East Africa Community Health (REACH) Policy Initiative in Uganda specifically. EVIPNet Africa includes African partners such as Burkina Faso, Cameroon, Central Africa Republic, Ethiopia, Mozambique, and Zambia [19].

This Clearinghouse is intended to facilitate timely access to decision-relevant information required by policymakers, stakeholders and researchers about the Ugandan health system and interventions. However, this resource could be limited by the adequacy of its content and the way it is organised. There is a lack of documented evidence on the available Uganda-specific health policy and systems-relevant documents that would inform whether most of the important documents have been included in the clearinghouse. Further, there is no clear framework to guide the organisation of the documents in the clearinghouse.

This paper provides a scoping review of Ugandaspecific health policy and systems-relevant documents produced in the last 15 years, up to December 2014. It is a step forward towards the mobilisation of documents for improving the Uganda Clearinghouse for Health Policy and Systems. The paper also identifies and provides a refined categorisation of the policy-relevant documents necessary for building the content of the clearinghouse to facilitate easy search by the users. The purpose of this paper is not just to indicate the ideal content of the Clearinghouse but to demonstrate that it is doable in a low-income setting. It aims to provide a framework which one can follow explicitly to generate an inventory of policy-relevant documents.

\section{Methods}

We reviewed published documents relevant for health policy and decision-making about the Uganda health system and interventions produced from January 1, 2000, to December 31, 2014. The year 2000 marked the beginning of implementation of key health sector reforms in Uganda [20]. Of interest was to identify and characterise documents produced since then to the beginning of this study. Our methods borrowed heavily from the 2005 Arksey and O'Malley methodological framework for scoping reviews [21].

\section{Step 1: Identification of the research question}

Since the research question guides the subsequent steps, including the search strategy, the Arksey and O'Malley 
methodological framework recommends considering all aspects of the research area to ensure a breadth of coverage and to define the relevant aspects of the research question [21]. In light of this, we developed our research questions as: What are the available types of documents relevant for health policy and systems that are specific to Uganda? What is the volume and nature (i.e. type, coverage of national priority areas, frequency of health-system topics) of these documents? From the onset, we were aware that such documents could be available as printed copies, published on websites of relevant non-governmental organisations (NGOs) and national institutions or just in the form of soft copies on personal computers that are not yet uploaded on websites. We focused on identifying and characterising the available documents relevant for health policy and systems published on websites.

\section{Step 2: Identification of relevant documents}

We conducted the search for Uganda-specific health policy and systems-relevant documents in January 2015. We selected the websites of relevant government institutions, international and national NGOs, health professional councils and associations, religious medical bureaus and research networks (Table 1). We used the search engine Google to locate such websites, which we then navigated by the tabs and menus available on the homepage (such as policy documents and guidelines, e-library, resources, publications, legislation). The fact that different websites are organised differently, we developed specific search strategies for each website depending on its individual navigability. In addition, we searched Google Scholar using the following keywords in various combinations with Boolean operators (and, or) [22]: Uganda, health policy, health system, guidelines, strategies, plans, and reports. We checked the reference lists of the documents found to expand our list of included documents. Importantly, we used the websites as an entry point to other repositories for national policy documents (Tables 2 and 3).

\section{Step 3: Screening and selection of relevant documents}

To minimise selection bias, two independent reviewers (BM and RB) screened all documents and selected those that were appropriate for our research question. Our selection involved the use of a pre-determined inclusion and exclusion criteria. We included all Uganda-specific published documents relevant to health policy and systems produced between 2000 and 2014. We borrowed from the Hoffman et al. [23] model shown in Fig. 1, which depicts the boundaries of health policy and systems research, to determine if documents were relevant to health policy and systems in order for us to include them. We therefore included all documents that addressed (1) issues related to health systems (i.e. on governance, financial, and delivery arrangements and implementation strategies); (2) policy about clinical issues that include essential medicines, diagnostics and medical supplies; and (3) policy about public/population issues such as policies on immunisation and family planning.

We excluded documents that (1) did not have national coverage (such as NGO project reports that covered only a few districts), (2) were at the draft stage, (3) covered less than a year (such as quarterly or semi-annual reports), or (4) described primary studies and systematic reviews.

\section{Step 4: Charting of the data}

The two independent reviewers used a specially developed data-charting form (Table 4) to extract data from each document on the title/topic, document type, coverage of national priority areas, coverage of health system topics, year published and the source of the document. We developed a tailored index of health policy documents based on the national priority issues, types of documents emerging from the search results and health system topics borrowed from the Health System Evidence [15, 24, 25]. We specifically categorised the documents as policies, strategies, plans, guidelines, rapid response summaries, and evidence briefs for policy, dialogue reports and other reports. We also coded the documents for national health priority areas as identified in the Second National Development Plan (NDPII 2015/ 16-2019/20) [24] and Second Health Policy (NHPII) [25]. The NDPII stipulates the Country's medium term strategic direction, priorities (including health priorities) and implementation strategies up to the year 2020. The national health priority areas are disease prevention, mitigation and control; health education, promotion, environmental health and nutrition; governance, coordination, monitoring and evaluation; maternal and child health; reproductive health; human resources for health; health financing; health infrastructure; early childhood development; essential medicines and supplies; palliative care services; rehabilitation services; and curative services $[24,25]$.

We further classified these documents as governance, financial and delivery arrangements, and as implementation strategies within the health systems [15]. The governance arrangements category includes documents on centralisation/decentralisation of health services, registration and accreditation of the services, consumer and stakeholder involvement in service delivery, stewardship of the non-state actors in health financing and delivery, among other topics [15]. The financial arrangements category includes documents on financing systems, funding organisations, remunerating providers, purchasing products and services, and incentives targeted at consumers [15]. The delivery arrangements category 
Table 1 Institutions/organisations whose websites were searched

\begin{tabular}{|c|c|}
\hline Institution/Organisation & Website \\
\hline \multicolumn{2}{|l|}{ International Organisations } \\
\hline 1. Centers for Disease Control and Prevention & http://www.cdc.gov/globalhealth/countries/uganda/ \\
\hline 2. Food and Agriculture Organization of the United Nations & http://www.fao.org/countryprofiles/index/en/?iso3=UGA \\
\hline 3. GAVI, the Vaccine Alliance & http://www.gavi.org/ \\
\hline 4. ICO Information Centre on HPV and Cancer & http://www.hpvcentre.net/ \\
\hline 5. United Nations Children's Fund & http://www.unicef.org/uganda/ \\
\hline 6. United Nations Population Fund & http://countryoffice.unfpa.org/uganda/ \\
\hline 7. United Nations Programme on HIV/AIDS & http://www.unaids.org/en/regionscountries/countries/uganda \\
\hline 8. United States Agency for International Development & https://www.usaid.gov/uganda \\
\hline 9. World Bank & http://www.worldbank.org/en/country/uganda \\
\hline 10. World Health Organization (WHO) & http://www.who.int/countries/uga/en/ \\
\hline \multicolumn{2}{|l|}{ Uganda Government Institutions } \\
\hline 1. Health Service Commission 2. Ministry of Agriculture & http://www.hsc.go.ug/ http://www.agriculture.go.ug/ \\
\hline 3. Ministry of Education and Sports & http://www.education.go.ug/ \\
\hline 4. Ministry of Finance, Planning and Economic Development & http://www.finance.go.ug/ \\
\hline 5. Ministry of Gender, Labour and Social Development & http://www.mglsd.go.ug/ \\
\hline 6. Ministry of Health & http://www.health.go.ug/ \\
\hline 7. National Council for Science and Technology & http://www.uncst.go.ug/ \\
\hline 8. National planning authority & http://npa.ug/ \\
\hline 9. Office of the Prime Minister & http://www.opm.go.ug/ \\
\hline 10. Parliament of Uganda & http://www.parliament.go.ug/new/ \\
\hline 11. Uganda AIDS Commission & http://www.aidsuganda.org/ \\
\hline 12. Uganda Bureau of Statistics & http://www.ubos.org/UNHS0910/home.html \\
\hline 13. Uganda Population Secretariat & http://popsec.org/ \\
\hline \multicolumn{2}{|l|}{ Locally Registered Non-Governmental Organizations } \\
\hline $\begin{array}{l}\text { 1. Elizabeth Glaser Pediatric AIDS Foundation } \\
\text { 2. Family Health International }\end{array}$ & $\begin{array}{l}\text { http://www.pedaids.org/countries/uganda } \\
\text { http://www.fhi360.org/countries/uganda }\end{array}$ \\
\hline 3. HEPS-Uganda & http://www.heps.or.ug/ \\
\hline 4. Infectious Diseases Institute & http://www.idi-makerere.com/ \\
\hline 5. Integrated Community Based Initiatives & http://www.icobi.or.ug/ \\
\hline 6. Joint Clinical Research Center & http://www.jcrc.org.ug/ \\
\hline 7. Plan International & https://plan-international.org/uganda \\
\hline 8. Population Reference Bureau & http://www.prb.org/Countries/Uganda.aspx \\
\hline 9. The AIDs Support Organization (TASO) & http://tasouganda.org/ \\
\hline 10. Uganda Community Based Health-Financing Association & http://ucbhfa.org/ \\
\hline 11. Uganda Women's Network & http://uwonet.or.ug/ \\
\hline 12. Women's International Cross Cultural Exchange & http://www.isis.or.ug/ \\
\hline \multicolumn{2}{|l|}{ Health Professional Councils And Associations } \\
\hline 1. Allied Health Practitioners Council & http://www.ahpc.ug/ \\
\hline $\begin{array}{l}\text { 2. Association of Surgeons and Association of Gynecologists and Obstetricians } \\
\text { of Uganda }\end{array}$ & http://sogc.org/aogu/ \\
\hline 3. Pharmaceutical society of Uganda & http://psu.or.ug/new/ \\
\hline 4. Uganda Dental Association & http://www.ugadent.org/ \\
\hline 5. Uganda Health Care Federation & http://ugandahealthcarefederation.blogspot.ug/ \\
\hline 6. Uganda Medical Association & http://www.uma.co.ug/ \\
\hline
\end{tabular}


Table 1 Institutions/organisations whose websites were searched (Continued)

\begin{tabular}{ll}
\hline 7. Uganda Medical and Dental Practitioner Health Council & http://www.umdpc.com/ \\
8. Uganda Nurses and Midwives Council & http://unmc.ug/ \\
Religious Medical Bureaus & \\
1. Uganda Catholic Medical Bureau & http://www.ucmb.co.ug/ \\
$\begin{array}{l}\text { 2. Uganda Protestant Medical Bureau } \\
\text { Academic Institutions/Research Networks }\end{array}$ & http://upmb.co.ug/ \\
1. EVIPNet & http://global.evipnet.org/ \\
2. Makerere University School of Public Health & http://www.musph.ac.ug/ \\
3. Uganda National Academy of Sciences & http://ugandanationalacademy.org/
\end{tabular}

covers documents on how care is designed to meet consumer needs, human resources for health, and support systems for the provision of care, plus where care is provided [15]. The implementation strategies category includes documents on consumer-, provider- and organisation-targeted strategies [15]. The third reviewer (EO) arbitrated areas that BM and RB disagreed on.

\section{Step 5: Collating, summarising and reporting results}

After charting the information from the evidence documents, we presented the review findings as numerical analyses of the volume of documents, their nature (i.e. type, coverage of national priority areas, the frequency of health system topics) and trends over time in the form of tables and charts. The data were summarised using descriptive statistics, including for the type of the documents included, the national health priority areas/ issues covered in the documents, the extent of coverage of the health priority for the different health policy and system domains, and the trends over time in the nature and distribution of the documents.

\section{Results}

The website hand-searches resulted in a total of 909 health policy and systems-relevant documents, including 10 duplicates. Of the remaining 899 documents, 265 met the selection criteria and were considered for analysis (Fig. 2).

\section{Volume of the documents and trends over time}

Most of the health policy and systems-relevant documents included in our review were rapid response summaries (18\%) and guidelines (13\%). The least were strategies (4\%) and policy dialogue reports (3\%) (Table 2). There was a consistent increase in the volume of the policy and system-relevant documents between 2000 and 2011 followed by a decline. The increase was from around six documents per year in 2000 to 49 per year in 2011 that later dropped to 27 in 2014 (Fig. 3).

\section{Coverage of the national health priority areas by the documents}

In Table 2, the top three national priority areas (clusters) addressed in the documents are governance, coordination, monitoring and evaluation $(74,28 \%)$, disease prevention, mitigation and control $(63,24 \%)$, and health education, promotion, environmental health and nutrition (41, 16\%). The least addressed national health priorities were curative services, health infrastructure, palliative care services, rehabilitation services, each addressed in three documents (1\%), and early childhood development (in only one document). The biggest percentage of policies (46\%), guidelines (31\%), policy dialogue reports $(29 \%)$ and evidence briefs for policy (18\%) addressed the cluster of disease prevention, mitigation and control, while most strategies (90\%) and rapid response summaries (17\%) covered the health education, promotion, environmental health and nutrition cluster, and plans (36\%) addressed the governance, coordination, monitoring and evaluation cluster (Table 2).

\section{Coverage of health systems topics by the documents}

Generally, the highest number of documents $(n=101$, $38 \%$ ) addressed the delivery arrangements domain, followed by the governance arrangements $(68,26 \%)$, implementation strategies $(56,21 \%)$, and lastly the financial arrangements $(16,6 \%)$ (Table 3$)$. Notably, the delivery arrangements domain was addressed by most of "other reports" (93\%), evidence briefs for policy (33\%) and rapid response summaries (25\%). Nearly, two-thirds (63\%) of guidelines, $49 \%$ of policies and $30 \%$ of rapid response summaries addressed the governance arrangements domain. A small percentage of evidence briefs for policy $(12 \%)$, rapid response summaries $(17 \%)$ and other reports $(5 \%)$ addressed the financial arrangement domain; this was not at all covered by guidelines, plans, policies, policy dialogue reports and strategies. Most of the plans (96\%) covered the implementation strategy; there was no single policy or other report that addressed it. Other documents that were about public or clinical issues 


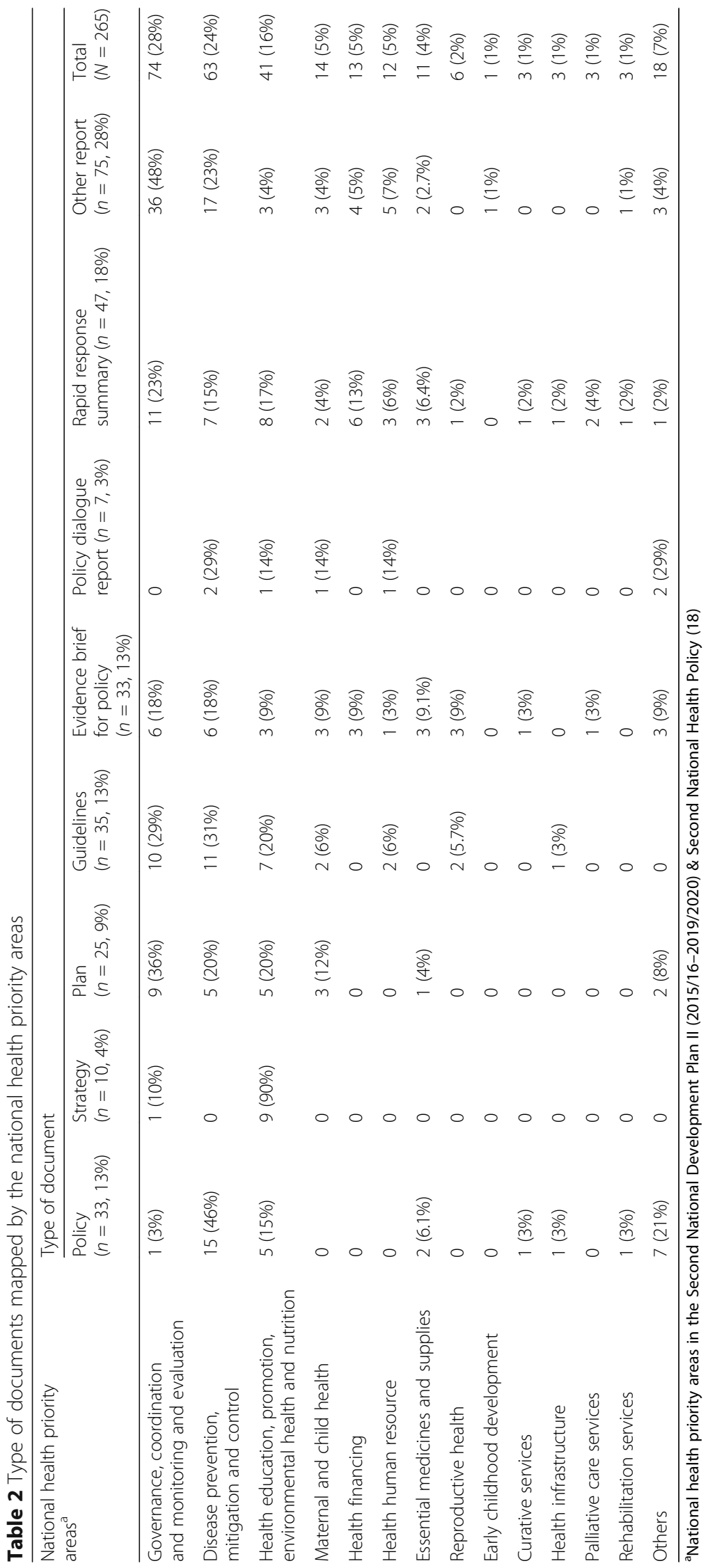




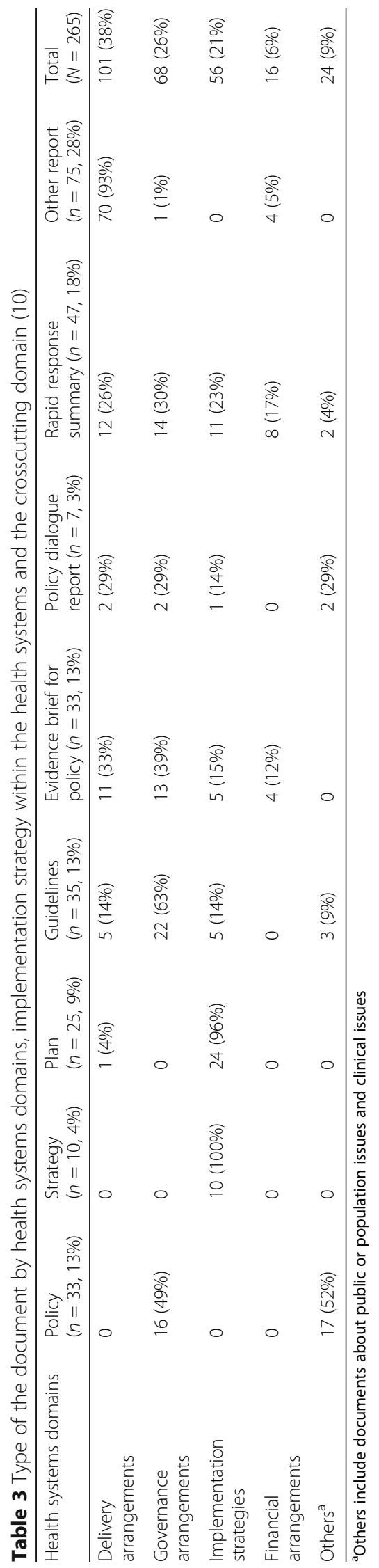




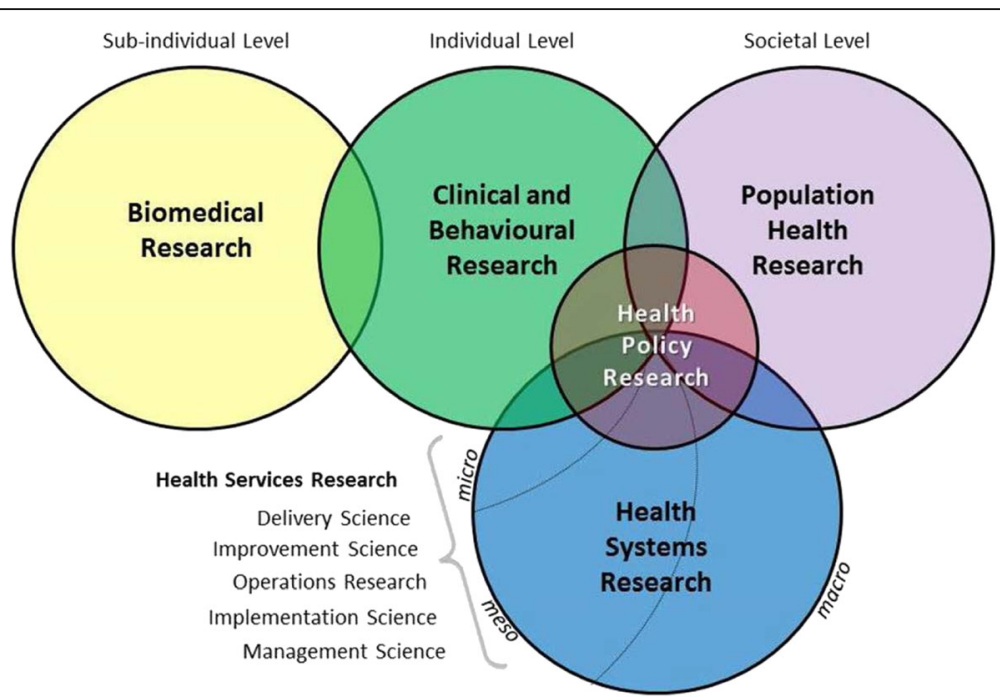

Fig. 1 Conceptual issues related to health systems research to inform a WHO Global Strategy on Health Systems Research. Adapted with permission from Hoffman et al. [20]

included $51 \%$ of policies, $29 \%$ of policy dialogue reports, $9 \%$ of guidelines and $4 \%$ of rapid response summaries.

\section{Discussion}

In this paper, we conducted a scoping review of policy and systems-relevant documents in Uganda to support the identification and characterisation of policy and systems-relevant documents for the content of an on-line repository. The first step of our framework, which is identification of documents, borrows from the Arksey and O'Malley methodological framework for scoping reviews. The second step involves the development of the tailored index of health policy documents based on the national priority issues, types of documents emerging from the search results and health system topics $[15,24,25]$.

A number of key findings emerged. First, the review demonstrates the availability of the policy and systemrelevant documents in the country that include policies, guidelines, plans, strategies, rapid response summaries, evidence briefs for policy, and policy dialogue reports. The available documents address several national health priority issues identified in the Second Health Policy and National Development Plan (2015/20162019/2020). Further, the review findings show varying coverage of the national health issues and health system topics by the documents, which is an important indication of areas of interest. Finally, it demonstrates that there has, until recently, been a progressive increase in the number of documents produced although the distribution of different document types has not been uniform.

\section{Findings in relation to other studies}

Our findings especially, on the availability of the policy and system-relevant documents are supported by literature from previous studies in low- and middle-income countries [26, 27]. We found out that apart from "other reports", rapid response summaries were by far the most dominant type of documents produced, although most of them were produced in a particular period, from 2008 to 2013. The fact that their production was majorly by research networks with a local presence $[18,19]$ is suggestive of increased local capacity to produce summaries. This may also reflect a growing interest by policymakers and stakeholders to use the summaries to address urgent policy questions in the country. This was corroborated in a study by Mijumbi, which showed that a rapid response mechanism service in Uganda has been widely used by national policymakers at the Ministry of Health and development partners and stakeholders from NGOs [26]. Although we utilised a multifaceted search strategy, relatively few plans, strategies and policy dialogue reports meeting the specified inclusion criteria were identified. The lack of these documents may indicate their scarcity in the country. This may instead point to the fact that these documents were not readily on governmental and NGOs' websites: an indication for lack of one-stop shops for knowledge sharing in the country. This is supported by findings from a study by Murphy [27], which demonstrated that information on training and deployment policies for health workers for maternal, newborn and child health in rural Africa was not available on governmental websites; it was instead readily located on the sites of institutions with a greater capacity for knowledge sharing. Generally, there was a noted 
Table 4 Documents reviewed

\begin{tabular}{|c|c|c|c|c|c|c|}
\hline $\mathrm{S} / \mathrm{N}$ & Title & $\begin{array}{l}\text { Type of } \\
\text { document }\end{array}$ & $\begin{array}{l}\text { National health } \\
\text { priority area }\end{array}$ & $\begin{array}{l}\text { Health systems } \\
\text { domains }\end{array}$ & Year & Source of the document \\
\hline 1 & $\begin{array}{l}\text { The Uganda Tuberculosis Communication } \\
\text { Strategy }\end{array}$ & Strategy & $\begin{array}{l}\text { Health education, } \\
\text { promotion, environmental } \\
\text { health \& nutrition }\end{array}$ & $\begin{array}{l}\text { Implementation } \\
\text { strategies }\end{array}$ & 2008 & Ministry of Health \\
\hline 2 & $\begin{array}{l}\text { National Couples HIV Counseling \& } \\
\text { Testing Communication Strategy }\end{array}$ & Strategy & $\begin{array}{l}\text { Health education, } \\
\text { promotion, environmental } \\
\text { health \& nutrition }\end{array}$ & $\begin{array}{l}\text { Implementation } \\
\text { strategies }\end{array}$ & 2009 & Ministry of Health \\
\hline 3 & $\begin{array}{l}\text { Nutrition in the National Child Survival } \\
\text { Strategy }\end{array}$ & Strategy & $\begin{array}{l}\text { Health education, } \\
\text { promotion, environmental } \\
\text { health \& nutrition }\end{array}$ & $\begin{array}{l}\text { Implementation } \\
\text { strategies }\end{array}$ & 2009 & Ministry of Health \\
\hline 4 & $\begin{array}{l}\text { Uganda National Communication Strategy } \\
\text { for Promoting Rational Use of Medicines }\end{array}$ & Strategy & $\begin{array}{l}\text { Health education, } \\
\text { promotion, environmental } \\
\text { health \& nutrition }\end{array}$ & $\begin{array}{l}\text { Implementation } \\
\text { strategies }\end{array}$ & 2009 & Ministry of Health \\
\hline 5 & $\begin{array}{l}\text { National Positive Living Communication } \\
\text { Strategy }\end{array}$ & Strategy & $\begin{array}{l}\text { Health education, } \\
\text { promotion, environmental } \\
\text { health \& nutrition }\end{array}$ & $\begin{array}{l}\text { Implementation } \\
\text { strategies }\end{array}$ & 2010 & Ministry of Health \\
\hline 6 & $\begin{array}{l}\text { Pediatric HIV Communication Campaign } \\
\text { Strategy }\end{array}$ & Strategy & $\begin{array}{l}\text { Health education, } \\
\text { promotion, environmental } \\
\text { health \& nutrition }\end{array}$ & $\begin{array}{l}\text { Implementation } \\
\text { strategies }\end{array}$ & 2010 & Ministry of Health \\
\hline 7 & $\begin{array}{l}\text { Integrating Population, Health and } \\
\text { Environment in Uganda }\end{array}$ & Strategy & $\begin{array}{l}\text { Health education, } \\
\text { promotion, environmental } \\
\text { health \& nutrition }\end{array}$ & $\begin{array}{l}\text { Implementation } \\
\text { strategies }\end{array}$ & 2009 & $\begin{array}{l}\text { Population Reference } \\
\text { Bureau }\end{array}$ \\
\hline 8 & $\begin{array}{l}\text { The National Advocacy Strategy } \\
\text { 2013-2022 }\end{array}$ & Strategy & $\begin{array}{l}\text { Health education, } \\
\text { promotion, environmental } \\
\text { health \& nutrition }\end{array}$ & $\begin{array}{l}\text { Implementation } \\
\text { strategies }\end{array}$ & 2013 & $\begin{array}{l}\text { Uganda Population } \\
\text { Secretariat }\end{array}$ \\
\hline 9 & WHO Country Cooperation Strategy & Strategy & $\begin{array}{l}\text { Governance, coordination } \\
\& \mathrm{M} \& \mathrm{E}\end{array}$ & $\begin{array}{l}\text { Implementation } \\
\text { strategies }\end{array}$ & 2009 & $\begin{array}{l}\text { World Health } \\
\text { Organization }\end{array}$ \\
\hline 10 & $\begin{array}{l}\text { National Household Water Treatment } \\
\text { and Safe Storage Strategies and Integrated } \\
\text { Household Environmental Health } \\
\text { Interventions }\end{array}$ & Strategy & $\begin{array}{l}\text { Health education, } \\
\text { promotion, environmental } \\
\text { health \& nutrition }\end{array}$ & $\begin{array}{l}\text { Implementation } \\
\text { strategies }\end{array}$ & 2011 & $\begin{array}{l}\text { World Health } \\
\text { Organization }\end{array}$ \\
\hline 11 & $\begin{array}{l}\text { Cost Effectiveness of Malaria Control } \\
\text { Programmes in Uganda: The Case Study } \\
\text { of Long Lasting Insecticide Treated Nets } \\
\text { (LLINs) and Indoor Residual Spraying }\end{array}$ & Report & Health financing & $\begin{array}{l}\text { Financial } \\
\text { arrangements }\end{array}$ & 2011 & $\begin{array}{l}\text { Economic Policy Research } \\
\text { Centre - Makerere } \\
\text { University Kampala }\end{array}$ \\
\hline 12 & $\begin{array}{l}\text { Situation Analysis to Determine the } \\
\text { Acceptability and Feasibility of Male } \\
\text { Circumcision Promotion in Uganda }\end{array}$ & Report & $\begin{array}{l}\text { Health education, } \\
\text { promotion, environmental } \\
\text { health \& nutrition }\end{array}$ & $\begin{array}{l}\text { Delivery } \\
\text { arrangements }\end{array}$ & 2007 & Family Health International \\
\hline 13 & $\begin{array}{l}\text { The Analysis of the Nutrition Situation } \\
\text { in Uganda }\end{array}$ & Report & $\begin{array}{l}\text { Health education, } \\
\text { promotion, environmental } \\
\text { health \& nutrition }\end{array}$ & $\begin{array}{l}\text { Delivery } \\
\text { arrangements }\end{array}$ & 2010 & FHI 360 \\
\hline 14 & Food and Nutrition Report & Report & $\begin{array}{l}\text { Health education, } \\
\text { promotion, environmental } \\
\text { health \& nutrition }\end{array}$ & $\begin{array}{l}\text { Delivery } \\
\text { arrangements }\end{array}$ & 2013 & $\begin{array}{l}\text { Food and Agriculture } \\
\text { Organization of the } \\
\text { United Nations }\end{array}$ \\
\hline 15 & $\begin{array}{l}\text { GAVI Alliance Progress Report for } \\
\text { Uganda, } 2007\end{array}$ & Report & $\begin{array}{l}\text { Disease prevention, } \\
\text { mitigation \& control }\end{array}$ & $\begin{array}{l}\text { Delivery } \\
\text { arrangements }\end{array}$ & 2007 & GAVI Alliance \\
\hline 16 & $\begin{array}{l}\text { Annual Report on Work in Crises } \\
\text { in Uganda }\end{array}$ & Report & $\begin{array}{l}\text { Human resources for } \\
\text { health }\end{array}$ & $\begin{array}{l}\text { Delivery } \\
\text { arrangements }\end{array}$ & 2007 & $\begin{array}{l}\text { Health Action in Crisis } \\
\text { (HAC) }\end{array}$ \\
\hline 17 & $\begin{array}{l}\text { Accessibility of Essential Medicines } \\
\text { and Diagnostics in Uganda }\end{array}$ & Report & $\begin{array}{l}\text { Governance, coordination } \\
\& \mathrm{M} \& \mathrm{E}\end{array}$ & $\begin{array}{l}\text { Delivery } \\
\text { arrangements }\end{array}$ & 2014 & HEPS Uganda \\
\hline 18 & Health Reforms in Uganda & Report & $\begin{array}{l}\text { Governance, coordination } \\
\& \text { M\&E }\end{array}$ & $\begin{array}{l}\text { Governance } \\
\text { arrangements }\end{array}$ & 2006 & $\begin{array}{l}\text { Institute of Public Health, } \\
\text { Makerere University, } \\
\text { Ministry of Health }\end{array}$ \\
\hline 19 & $\begin{array}{l}\text { Millennium Development Goals, } \\
\text { Progress Report }\end{array}$ & Report & $\begin{array}{l}\text { Disease prevention, } \\
\text { mitigation \& control }\end{array}$ & $\begin{array}{l}\text { Delivery } \\
\text { arrangements }\end{array}$ & 2013 & $\begin{array}{l}\text { Ministry of Finance, } \\
\text { Planning and Economic } \\
\text { Development }\end{array}$ \\
\hline
\end{tabular}


Table 4 Documents reviewed (Continued)

\begin{tabular}{|c|c|c|c|c|c|c|}
\hline 20 & The State of Uganda Population & Report & $\begin{array}{l}\text { Governance, coordination } \\
\& \text { M\&E }\end{array}$ & $\begin{array}{l}\text { Delivery } \\
\text { arrangements }\end{array}$ & 2013 & $\begin{array}{l}\text { Ministry of Finance, } \\
\text { Planning and Economic } \\
\text { Development }\end{array}$ \\
\hline 21 & $\begin{array}{l}\text { Millennium Development Goals, } \\
\text { Progress Report }\end{array}$ & Report & $\begin{array}{l}\text { Disease prevention, } \\
\text { mitigation \& control }\end{array}$ & $\begin{array}{l}\text { Delivery } \\
\text { arrangements }\end{array}$ & 2010 & $\begin{array}{l}\text { Ministry of Finance, } \\
\text { Planning and Economic } \\
\text { Development }\end{array}$ \\
\hline 22 & National Health Accounts Report & Report & Health financing & $\begin{array}{l}\text { Financial } \\
\text { arrangements }\end{array}$ & 2008 & Ministry of Health \\
\hline 23 & Health Financing Review & Report & Health financing & $\begin{array}{l}\text { Financial } \\
\text { arrangements }\end{array}$ & 2010 & Ministry of Health \\
\hline 24 & $\begin{array}{l}\text { Final Report Essential Medicines and } \\
\text { Health Supplies Tracking Study }\end{array}$ & Report & $\begin{array}{l}\text { Disease prevention, } \\
\text { mitigation \& control }\end{array}$ & $\begin{array}{l}\text { Delivery } \\
\text { arrangements }\end{array}$ & 2009 & Ministry of Health \\
\hline 25 & Report Malaria Indicator Survey & Report & $\begin{array}{l}\text { Governance, coordination } \\
\& \text { M\&E }\end{array}$ & $\begin{array}{l}\text { Delivery } \\
\text { arrangements }\end{array}$ & 2009 & Ministry of Health \\
\hline 26 & $\begin{array}{l}\text { Situation Analysis Village Health Teams } \\
\text { Uganda } 2009\end{array}$ & Report & $\begin{array}{l}\text { Human resources } \\
\text { for health }\end{array}$ & $\begin{array}{l}\text { Delivery } \\
\text { arrangements }\end{array}$ & 2009 & Ministry of Health \\
\hline 27 & $\begin{array}{l}\text { Status of Antiretroviral Therapy Service } \\
\text { Delivery in Uganda }\end{array}$ & Report & $\begin{array}{l}\text { Disease prevention, } \\
\text { mitigation \& control }\end{array}$ & $\begin{array}{l}\text { Delivery } \\
\text { arrangements }\end{array}$ & 2010 & Ministry of Health \\
\hline 28 & $\begin{array}{l}\text { HIV Sero Behavioral Survey in Fishing } \\
\text { Communities of the Lake Victoria Basin } \\
\text { of Uganda }\end{array}$ & Report & $\begin{array}{l}\text { Disease prevention, } \\
\text { mitigation \& control }\end{array}$ & $\begin{array}{l}\text { Delivery } \\
\text { arrangements }\end{array}$ & 2011 & Ministry of Health \\
\hline 29 & Uganda Malaria Country profile & Report & $\begin{array}{l}\text { Governance, coordination } \\
\& M \& E\end{array}$ & $\begin{array}{l}\text { Delivery } \\
\text { arrangements }\end{array}$ & 2011 & Ministry of Health \\
\hline 30 & $\begin{array}{l}\text { Annual Health Sector Performance } \\
\text { Report }\end{array}$ & Report & $\begin{array}{l}\text { Governance, coordination } \\
\& M \& E\end{array}$ & $\begin{array}{l}\text { Delivery } \\
\text { arrangements }\end{array}$ & 2013 & Ministry of Health \\
\hline 31 & National Performance Report & Report & $\begin{array}{l}\text { Governance, coordination } \\
\& M \& E\end{array}$ & $\begin{array}{l}\text { Delivery } \\
\text { arrangements }\end{array}$ & 2013 & Ministry of Health \\
\hline 32 & $\begin{array}{l}\text { Mid Term Review of the 2010-2015 } \\
\text { Malaria Strategic Plan }\end{array}$ & Report & $\begin{array}{l}\text { Governance, coordination } \\
\& M \& E\end{array}$ & $\begin{array}{l}\text { Delivery } \\
\text { arrangements }\end{array}$ & 2014 & $\begin{array}{l}\text { National Malaria } \\
\text { Control Programme }\end{array}$ \\
\hline 33 & $\begin{array}{l}\text { Gender Responsive Indicators for } \\
\text { Sectors - Final Report }\end{array}$ & Report & Others & $\begin{array}{l}\text { Delivery } \\
\text { arrangements }\end{array}$ & 2012 & $\begin{array}{l}\text { National Planning } \\
\text { Authority }\end{array}$ \\
\hline 34 & $\begin{array}{l}\text { HPV Vaccination in Africa: Lessons } \\
\text { Learned from a Pilot Program in } \\
\text { Uganda }\end{array}$ & Report & $\begin{array}{l}\text { Disease prevention, } \\
\text { mitigation \& control }\end{array}$ & $\begin{array}{l}\text { Delivery } \\
\text { arrangements }\end{array}$ & 2000 & PATH \\
\hline 35 & $\begin{array}{l}\text { National Capacity Assessment of Public } \\
\text { and Private Institutions Involved in } \\
\text { HIV/AIDS Service Delivery }\end{array}$ & Report & $\begin{array}{l}\text { Governance, coordination } \\
\& M \& E\end{array}$ & $\begin{array}{l}\text { Delivery } \\
\text { arrangements }\end{array}$ & 2009 & $\begin{array}{l}\text { Uganda AIDS } \\
\text { Commission }\end{array}$ \\
\hline 36 & $\begin{array}{l}\text { PLHIV Sigma Index Report - Uganda } \\
\text { Country Assessment 2013-1 }\end{array}$ & Report & $\begin{array}{l}\text { Disease prevention, } \\
\text { mitigation \& control }\end{array}$ & $\begin{array}{l}\text { Delivery } \\
\text { arrangements }\end{array}$ & 2013 & $\begin{array}{l}\text { Uganda AIDS } \\
\text { Commission }\end{array}$ \\
\hline 37 & $\begin{array}{l}\text { Uganda HIV Country Progress Report, } \\
2013\end{array}$ & Report & $\begin{array}{l}\text { Disease prevention, } \\
\text { mitigation \& control }\end{array}$ & $\begin{array}{l}\text { Delivery } \\
\text { arrangements }\end{array}$ & 2013 & $\begin{array}{l}\text { Uganda AIDS } \\
\text { Commission }\end{array}$ \\
\hline 38 & $\begin{array}{l}\text { High PMTCT Program Uptake and } \\
\text { Coverage of Mothers, Their Partners, } \\
\text { and Babies in Northern Uganda: } \\
\text { Achievements and Lessons Learned } \\
\text { Over } 10 \text { Years of Implementation } \\
\text { (2002-2011) }\end{array}$ & Report & $\begin{array}{l}\text { Disease prevention, } \\
\text { mitigation \& control }\end{array}$ & $\begin{array}{l}\text { Delivery } \\
\text { arrangements }\end{array}$ & 2014 & $\begin{array}{l}\text { Uganda AIDS } \\
\text { Commission }\end{array}$ \\
\hline 39 & $\begin{array}{l}\text { Progress in the Fight Against HIV and } \\
\text { AIDS, } 2014\end{array}$ & Report & $\begin{array}{l}\text { Disease prevention, } \\
\text { mitigation \& control }\end{array}$ & $\begin{array}{l}\text { Delivery } \\
\text { arrangements }\end{array}$ & 2014 & $\begin{array}{l}\text { Uganda AIDS } \\
\text { Commission }\end{array}$ \\
\hline 40 & Children and HIV/AIDS Key Statistics & Report & $\begin{array}{l}\text { Disease prevention, } \\
\text { mitigation \& control }\end{array}$ & $\begin{array}{l}\text { Delivery } \\
\text { arrangements }\end{array}$ & 2002 & $\begin{array}{l}\text { Uganda AIDS } \\
\text { Commission }\end{array}$ \\
\hline 41 & $\begin{array}{l}\text { Final Report for Midterm Evaluation of the } \\
\text { Project Comprehensive HIV/AIDS Prevention } \\
\text { Among Fishing Communities on Lakes } \\
\text { George and Edward Project (CHAPFICO) }\end{array}$ & Report & $\begin{array}{l}\text { Disease prevention, } \\
\text { mitigation \& control }\end{array}$ & $\begin{array}{l}\text { Delivery } \\
\text { arrangements }\end{array}$ & 2002 & $\begin{array}{l}\text { Uganda AIDS } \\
\text { Commission }\end{array}$ \\
\hline 42 & $\begin{array}{l}\text { Uganda Demographic and Health Survey } \\
\text { 2000-2001 }\end{array}$ & Report & $\begin{array}{l}\text { Governance, coordination } \\
\text { \& M\&E }\end{array}$ & $\begin{array}{l}\text { Delivery } \\
\text { arrangements }\end{array}$ & 2000 & $\begin{array}{l}\text { Uganda Bureau } \\
\text { of Statistics }\end{array}$ \\
\hline
\end{tabular}


Table 4 Documents reviewed (Continued)

\begin{tabular}{|c|c|c|c|c|c|}
\hline $\begin{array}{l}43 \text { Uganda National Household Survey } \\
\text { 2002/2003 }\end{array}$ & Report & $\begin{array}{l}\text { Governance, coordination } \\
\text { \& M\&E }\end{array}$ & $\begin{array}{l}\text { Delivery } \\
\text { arrangements }\end{array}$ & 2002 & $\begin{array}{l}\text { Uganda Bureau } \\
\text { of Statistics }\end{array}$ \\
\hline $\begin{array}{l}\text { Uganda HIV/AIDS Sero-behavioural } \\
\text { Survey }\end{array}$ & Report & $\begin{array}{l}\text { Disease prevention, } \\
\text { mitigation \& control }\end{array}$ & $\begin{array}{l}\text { Delivery } \\
\text { arrangements }\end{array}$ & 2004 & $\begin{array}{l}\text { Uganda Bureau } \\
\text { of Statistics }\end{array}$ \\
\hline $\begin{array}{l}\text { Uganda National Household Survey } \\
\text { 2005/2006 }\end{array}$ & Report & $\begin{array}{l}\text { Governance, coordination } \\
\& M \& E\end{array}$ & $\begin{array}{l}\text { Delivery } \\
\text { arrangements }\end{array}$ & 2005 & $\begin{array}{l}\text { Uganda Bureau } \\
\text { of Statistics }\end{array}$ \\
\hline A Demographic and Health Survey 2006 & Report & $\begin{array}{l}\text { Governance, coordination } \\
\& \text { M\&E }\end{array}$ & $\begin{array}{l}\text { Delivery } \\
\text { arrangements }\end{array}$ & 2006 & $\begin{array}{l}\text { Uganda Bureau } \\
\text { of Statistics }\end{array}$ \\
\hline $\begin{array}{l}47 \text { Uganda Bureau of Statistics Annual Report } \\
\text { 2008/2009 }\end{array}$ & Report & $\begin{array}{l}\text { Governance, coordination } \\
\& \text { M\&E }\end{array}$ & $\begin{array}{l}\text { Delivery } \\
\text { arrangements }\end{array}$ & 2008 & $\begin{array}{l}\text { Uganda Bureau } \\
\text { of Statistics }\end{array}$ \\
\hline $\begin{array}{l}\text { Uganda - Gender Based Violence Survey, } \\
2009\end{array}$ & Report & Others & $\begin{array}{l}\text { Delivery } \\
\text { arrangements }\end{array}$ & 2009 & $\begin{array}{l}\text { Uganda Bureau } \\
\text { of Statistics }\end{array}$ \\
\hline $\begin{array}{l}\text { Uganda Bureau of Statistics Annual Report } \\
\text { 2009/2010 }\end{array}$ & Report & $\begin{array}{l}\text { Governance, coordination } \\
\& \text { M\&E }\end{array}$ & $\begin{array}{l}\text { Delivery } \\
\text { arrangements }\end{array}$ & 2009 & $\begin{array}{l}\text { Uganda Bureau } \\
\text { of Statistics }\end{array}$ \\
\hline Uganda National Household Survey & Report & $\begin{array}{l}\text { Governance, coordination } \\
\& M \& E\end{array}$ & $\begin{array}{l}\text { Delivery } \\
\text { arrangements }\end{array}$ & 2009 & $\begin{array}{l}\text { Uganda Bureau } \\
\text { of Statistics }\end{array}$ \\
\hline $\begin{array}{l}51 \text { Uganda National Household Survey } \\
\text { 2009/2010 }\end{array}$ & Report & $\begin{array}{l}\text { Governance, coordination } \\
\& \text { M\&E }\end{array}$ & $\begin{array}{l}\text { Delivery } \\
\text { arrangements }\end{array}$ & 2009 & $\begin{array}{l}\text { Uganda Bureau } \\
\text { of Statistics }\end{array}$ \\
\hline 52 UNHS 2009/2010 Socio-Economic Report & Report & $\begin{array}{l}\text { Governance, coordination } \\
\& M \& E\end{array}$ & $\begin{array}{l}\text { Delivery } \\
\text { arrangements }\end{array}$ & 2009 & $\begin{array}{l}\text { Uganda Bureau } \\
\text { of Statistics }\end{array}$ \\
\hline $\begin{array}{l}3 \text { Uganda Bureau of Statistics Annual Report } \\
\text { 2010/2011 }\end{array}$ & Report & $\begin{array}{l}\text { Governance, coordination } \\
\& \text { M\&E }\end{array}$ & $\begin{array}{l}\text { Delivery } \\
\text { arrangements }\end{array}$ & 2010 & $\begin{array}{l}\text { Uganda Bureau } \\
\text { of Statistics }\end{array}$ \\
\hline $\begin{array}{l}54 \text { Uganda Bureau of Statistics Annual Report } \\
\text { 2011/2012 }\end{array}$ & Report & $\begin{array}{l}\text { Governance, coordination } \\
\& \text { M\&E }\end{array}$ & $\begin{array}{l}\text { Delivery } \\
\text { arrangements }\end{array}$ & 2011 & $\begin{array}{l}\text { Uganda Bureau } \\
\text { of Statistics }\end{array}$ \\
\hline Uganda Demographic and Health Survey & Report & $\begin{array}{l}\text { Governance, coordination } \\
\text { \& M\&E }\end{array}$ & $\begin{array}{l}\text { Delivery } \\
\text { arrangements }\end{array}$ & 2011 & $\begin{array}{l}\text { Uganda Bureau } \\
\text { of Statistics }\end{array}$ \\
\hline $\begin{array}{l}56 \text { Statistical Abstract - A Summary on Various } \\
\text { Socio-Economic Indicators in Uganda }\end{array}$ & Report & $\begin{array}{l}\text { Governance, coordination } \\
\& \text { M\&E }\end{array}$ & $\begin{array}{l}\text { Delivery } \\
\text { arrangements }\end{array}$ & 2012 & $\begin{array}{l}\text { Uganda Bureau } \\
\text { of Statistics }\end{array}$ \\
\hline $\begin{array}{l}57 \text { Uganda Bureau of Statistics Annual Report } \\
\text { 2012/2013 }\end{array}$ & Report & $\begin{array}{l}\text { Governance, coordination } \\
\& \text { M\&E }\end{array}$ & $\begin{array}{l}\text { Delivery } \\
\text { arrangements }\end{array}$ & 2012 & $\begin{array}{l}\text { Uganda Bureau } \\
\text { of Statistics }\end{array}$ \\
\hline $\begin{array}{l}58 \text { Statistical Abstract - A Summary on Various } \\
\text { Socio-Economic Indicators in Uganda }\end{array}$ & Report & $\begin{array}{l}\text { Governance, coordination } \\
\& \text { M\&E }\end{array}$ & $\begin{array}{l}\text { Delivery } \\
\text { arrangements }\end{array}$ & 2013 & $\begin{array}{l}\text { Uganda Bureau } \\
\text { of Statistics }\end{array}$ \\
\hline $\begin{array}{l}59 \text { Report on Community Health Financing in } \\
\text { Uganda }\end{array}$ & Report & $\begin{array}{l}\text { Governance, coordination } \\
\& \text { M\&E }\end{array}$ & $\begin{array}{l}\text { Delivery } \\
\text { arrangements }\end{array}$ & 2009 & $\begin{array}{l}\text { Uganda Community } \\
\text { Based Health Financing } \\
\text { Association }\end{array}$ \\
\hline $\begin{array}{l}50 \text { Health Service Commission Guidelines for } \\
\text { the Recruitment of Health Workers in } \\
\text { Districts and Urban Authorities } 2005\end{array}$ & Report & $\begin{array}{l}\text { Human resources for } \\
\text { health }\end{array}$ & $\begin{array}{l}\text { Delivery } \\
\text { arrangements }\end{array}$ & 2005 & $\begin{array}{l}\text { Uganda Health Service } \\
\text { Commission }\end{array}$ \\
\hline $\begin{array}{l}61 \text { Health Service Commission Annual } \\
\text { Report 2008/2009 }\end{array}$ & Report & $\begin{array}{l}\text { Human resources for } \\
\text { health }\end{array}$ & $\begin{array}{l}\text { Delivery } \\
\text { arrangements }\end{array}$ & 2008 & $\begin{array}{l}\text { Uganda Health Service } \\
\text { Commission }\end{array}$ \\
\hline $\begin{array}{l}62 \text { Health Service Commission Annual } \\
\text { Report 2009-2010 }\end{array}$ & Report & $\begin{array}{l}\text { Human resources for } \\
\text { health }\end{array}$ & $\begin{array}{l}\text { Delivery } \\
\text { arrangements }\end{array}$ & 2009 & $\begin{array}{l}\text { Uganda Health Service } \\
\text { Commission }\end{array}$ \\
\hline 63 Uganda Malaria Program review Report & Report & $\begin{array}{l}\text { Disease prevention, } \\
\text { mitigation \& control }\end{array}$ & $\begin{array}{l}\text { Delivery } \\
\text { arrangements }\end{array}$ & 2001 & $\begin{array}{l}\text { Uganda Malaria Control } \\
\text { Programme }\end{array}$ \\
\hline $\begin{array}{l}64 \text { The Status of Implementation of the } \\
\text { Education Sector Early Childhood } \\
\text { Development Policy in Uganda }\end{array}$ & Report & $\begin{array}{l}\text { Early childhood } \\
\text { development }\end{array}$ & $\begin{array}{l}\text { Delivery } \\
\text { arrangements }\end{array}$ & 2012 & $\begin{array}{l}\text { Uganda Ministry of } \\
\text { Education and Sports }\end{array}$ \\
\hline $\begin{array}{l}65 \text { State of Uganda Population Report } \\
2006\end{array}$ & Report & $\begin{array}{l}\text { Governance, coordination } \\
\& \text { M\&E }\end{array}$ & $\begin{array}{l}\text { Delivery } \\
\text { arrangements }\end{array}$ & 2006 & $\begin{array}{l}\text { Uganda Population } \\
\text { Secretariat }\end{array}$ \\
\hline $\begin{array}{l}56 \text { State of Uganda Population Report } \\
2007\end{array}$ & Report & $\begin{array}{l}\text { Governance, coordination } \\
\& \text { M\&E }\end{array}$ & $\begin{array}{l}\text { Delivery } \\
\text { arrangements }\end{array}$ & 2007 & $\begin{array}{l}\text { Uganda Population } \\
\text { Secretariat }\end{array}$ \\
\hline $\begin{array}{l}\text { State of Uganda Population Report } \\
2008\end{array}$ & Report & $\begin{array}{l}\text { Governance, coordination } \\
\& \text { M\&E }\end{array}$ & $\begin{array}{l}\text { Delivery } \\
\text { arrangements }\end{array}$ & 2008 & $\begin{array}{l}\text { Uganda Population } \\
\text { Secretariat }\end{array}$ \\
\hline $\begin{array}{l}\text { State of Uganda Population Report } \\
2009\end{array}$ & Report & $\begin{array}{l}\text { Governance, coordination } \\
\& \text { M\&E }\end{array}$ & $\begin{array}{l}\text { Delivery } \\
\text { arrangements }\end{array}$ & 2009 & $\begin{array}{l}\text { Uganda Population } \\
\text { Secretariat }\end{array}$ \\
\hline
\end{tabular}


Table 4 Documents reviewed (Continued)

\begin{tabular}{|c|c|c|c|c|c|c|}
\hline 69 & $\begin{array}{l}\text { State of Uganda Population Report } \\
2010\end{array}$ & Report & $\begin{array}{l}\text { Governance, coordination } \\
\& \text { M\&E }\end{array}$ & $\begin{array}{l}\text { Delivery } \\
\text { arrangements }\end{array}$ & 2010 & $\begin{array}{l}\text { Uganda Population } \\
\text { Secretariat }\end{array}$ \\
\hline 70 & $\begin{array}{l}\text { State of Uganda Population Report } \\
2011\end{array}$ & Report & $\begin{array}{l}\text { Governance, coordination } \\
\& \text { M\&E }\end{array}$ & $\begin{array}{l}\text { Delivery } \\
\text { arrangements }\end{array}$ & 2011 & $\begin{array}{l}\text { Uganda Population } \\
\text { Secretariat }\end{array}$ \\
\hline 71 & $\begin{array}{l}\text { State of Uganda Population Report } \\
2013\end{array}$ & Report & $\begin{array}{l}\text { Governance, coordination } \\
\& \text { M\&E }\end{array}$ & $\begin{array}{l}\text { Delivery } \\
\text { arrangements }\end{array}$ & 2013 & $\begin{array}{l}\text { Uganda Population } \\
\text { Secretariat }\end{array}$ \\
\hline 72 & $\begin{array}{l}\text { International Conference on Population } \\
\text { and Development (ICPD) Beyond } 2014 \\
\text { Review Uganda Country Report }\end{array}$ & Report & $\begin{array}{l}\text { Governance, coordination } \\
\& M \& E\end{array}$ & $\begin{array}{l}\text { Delivery } \\
\text { arrangements }\end{array}$ & 2014 & $\begin{array}{l}\text { Uganda Population } \\
\text { Secretariat }\end{array}$ \\
\hline 73 & $\begin{array}{l}\text { State of Uganda Population Report } \\
2014\end{array}$ & Report & $\begin{array}{l}\text { Governance, coordination } \\
\& M \& E\end{array}$ & $\begin{array}{l}\text { Delivery } \\
\text { arrangements }\end{array}$ & 2014 & $\begin{array}{l}\text { Uganda Population } \\
\text { Secretariat }\end{array}$ \\
\hline 74 & $\begin{array}{l}\text { HIV Prevention Response and Modes } \\
\text { of Transmission Analysis - Uganda }\end{array}$ & Report & $\begin{array}{l}\text { Disease prevention, } \\
\text { mitigation \& control }\end{array}$ & $\begin{array}{l}\text { Delivery } \\
\text { arrangements }\end{array}$ & 2009 & UNAIDS \\
\hline 75 & Uganda UNGASS Report for 2013 & Report & $\begin{array}{l}\text { Disease prevention, } \\
\text { mitigation \& control }\end{array}$ & $\begin{array}{l}\text { Delivery } \\
\text { arrangements }\end{array}$ & 2013 & UNAIDS \\
\hline 76 & State of Uganda Population report & Report & $\begin{array}{l}\text { Governance, coordination } \\
\& M \& E\end{array}$ & $\begin{array}{l}\text { Delivery } \\
\text { arrangements }\end{array}$ & 2010 & UNFPA \\
\hline 77 & UGANDA ANNUAL REPORT UNICEF & Report & Maternal and child health & $\begin{array}{l}\text { Delivery } \\
\text { arrangements }\end{array}$ & 2006 & UNICEF \\
\hline 78 & UNICEF - Uganda 2012 Statement & Report & Maternal and child health & $\begin{array}{l}\text { Delivery } \\
\text { arrangements }\end{array}$ & 2012 & UNICEF \\
\hline 79 & UNICEF Country Office Annual Report & Report & Maternal and child health & $\begin{array}{l}\text { Delivery } \\
\text { arrangements }\end{array}$ & 2013 & UNICEF \\
\hline 80 & $\begin{array}{l}\text { Situational Analysis on the Rights if } \\
\text { Children with Disabilities in Uganda }\end{array}$ & Report & Rehabilitation services & $\begin{array}{l}\text { Delivery } \\
\text { arrangements }\end{array}$ & 2014 & UNICEF \\
\hline 81 & Uganda Pharmaceutical Sector Report & Report & $\begin{array}{l}\text { Essential medicines \& } \\
\text { supplies }\end{array}$ & $\begin{array}{l}\text { Delivery } \\
\text { arrangements }\end{array}$ & 2010 & USAID \\
\hline 82 & Working Paper & Report & Health financing & $\begin{array}{l}\text { Financial } \\
\text { arrangements }\end{array}$ & 2010 & World bank \\
\hline 83 & $\begin{array}{l}\text { Uganda - Quantitative Service } \\
\text { Delivery Survey in Health } 2000\end{array}$ & Report & $\begin{array}{l}\text { Governance, coordination } \\
\& \text { M\&E }\end{array}$ & $\begin{array}{l}\text { Delivery } \\
\text { arrangements }\end{array}$ & 2000 & World Bank \\
\hline 84 & Well-Being of Older People Survey & Report & Others & $\begin{array}{l}\text { Delivery } \\
\text { arrangements }\end{array}$ & 2009 & $\begin{array}{l}\text { World Health } \\
\text { Organization }\end{array}$ \\
\hline 85 & $\begin{array}{l}\text { Report on Specifications for } \\
\text { Pharmaceutical Preparation }\end{array}$ & Report & $\begin{array}{l}\text { Essential medicines } \\
\& \text { supplies }\end{array}$ & $\begin{array}{l}\text { Delivery } \\
\text { arrangements }\end{array}$ & 2014 & $\begin{array}{l}\text { World Health } \\
\text { Organization }\end{array}$ \\
\hline 86 & $\begin{array}{l}\text { How Safe is the Practice of } \\
\text { Reflexology? }\end{array}$ & $\begin{array}{l}\text { Rapid } \\
\text { response } \\
\text { summary }\end{array}$ & Rehabilitation services & $\begin{array}{l}\text { Governance } \\
\text { arrangements }\end{array}$ & 2010 & $\begin{array}{l}\text { SURE Project - Makerere } \\
\text { University College of } \\
\text { Health Sciences }\end{array}$ \\
\hline 87 & $\begin{array}{l}\text { What are the Best Methods for } \\
\text { Involving Patients in Health System } \\
\text { Decision-Making in Uganda? }\end{array}$ & $\begin{array}{l}\text { Rapid } \\
\text { response } \\
\text { summary }\end{array}$ & $\begin{array}{l}\text { Governance, coordination } \\
\text { \& M\&E }\end{array}$ & $\begin{array}{l}\text { Governance } \\
\text { arrangements }\end{array}$ & 2010 & $\begin{array}{l}\text { SURE Project - Makerere } \\
\text { University College of } \\
\text { Health Sciences }\end{array}$ \\
\hline 88 & $\begin{array}{l}\text { What Does Policy Implementation } \\
\text { Monitoring Entail? }\end{array}$ & $\begin{array}{l}\text { Rapid } \\
\text { response } \\
\text { summary }\end{array}$ & $\begin{array}{l}\text { Governance, coordination } \\
\& \text { M\&E }\end{array}$ & $\begin{array}{l}\text { Governance } \\
\text { arrangements }\end{array}$ & 2010 & $\begin{array}{l}\text { SURE Project - Makerere } \\
\text { University College of } \\
\text { Health Sciences }\end{array}$ \\
\hline 89 & $\begin{array}{l}\text { Are Herbal Photolarvicides Efficient and } \\
\text { Safe to Use in Vector Management? }\end{array}$ & $\begin{array}{l}\text { Rapid } \\
\text { response } \\
\text { summary }\end{array}$ & $\begin{array}{l}\text { Health education, } \\
\text { promotion, environmental } \\
\text { health \& nutrition }\end{array}$ & $\begin{array}{l}\text { Governance } \\
\text { arrangements }\end{array}$ & 2011 & $\begin{array}{l}\text { SURE Project - Makerere } \\
\text { University College of } \\
\text { Health Sciences }\end{array}$ \\
\hline 90 & $\begin{array}{l}\text { Can Decentralization of Health Services } \\
\text { Improve Health Service Delivery in } \\
\text { Uganda? }\end{array}$ & $\begin{array}{l}\text { Rapid } \\
\text { response } \\
\text { summary }\end{array}$ & $\begin{array}{l}\text { Governance, coordination } \\
\& \text { M\&E }\end{array}$ & $\begin{array}{l}\text { Governance } \\
\text { arrangements }\end{array}$ & 2011 & $\begin{array}{l}\text { SURE Project - Makerere } \\
\text { University College of } \\
\text { Health Sciences }\end{array}$ \\
\hline 91 & $\begin{array}{l}\text { Does Introducing Deliveries at Health } \\
\text { Center II Improve Maternal Outcomes? }\end{array}$ & $\begin{array}{l}\text { Rapid } \\
\text { response } \\
\text { summary }\end{array}$ & Health infrastructure & $\begin{array}{l}\text { Governance } \\
\text { arrangements }\end{array}$ & 2011 & $\begin{array}{l}\text { SURE Project - Makerere } \\
\text { University College of } \\
\text { Health Sciences }\end{array}$ \\
\hline 92 & $\begin{array}{l}\text { Knowledge Management: How Can } \\
\text { Policy Makers Improve the Use of } \\
\text { Data in Policy Discussions and }\end{array}$ & $\begin{array}{l}\text { Rapid } \\
\text { response } \\
\text { summary }\end{array}$ & $\begin{array}{l}\text { Governance, coordination } \\
\& \text { M\&E }\end{array}$ & $\begin{array}{l}\text { Governance } \\
\text { arrangements }\end{array}$ & 2011 & $\begin{array}{l}\text { SURE Project - Makerere } \\
\text { University College of } \\
\text { Health Sciences }\end{array}$ \\
\hline
\end{tabular}


Table 4 Documents reviewed (Continued)

93 Monitoring of Medicines in Health Systems

94 Stem Cell Policies and Regulations Globally - An Overview of the Content and Context

95 What Are the Options for Re-Centralization of the Health Sector in Uganda?

96 What is Involved in the Efficient Relationship between the Ministry of Health and Teaching Hospitals in Order for both Institutions to Effectively Meet their Objectives?

97 What Role Can Regional Tear Play in Facilitating Health Service Delivery

98 What Risks May Food Vendors in Urban Areas Expose the Ugandan Population To?

99 Uganda EPI Vertical vs Integrated Approach

100 Cost Analysis Tool for Fistula Repair

101 Appropriate Health Financing Strategies for Uganda

102 Diagnosis Cost of a New Sputum Smear Positive TB Case in Children in Low Income Countries

103 Social Health Insurance \& Improvement of Health Systems Organization and Utilization of Services

104 Management of (Expensive) Medical Equipment; Lessons from Other Countries

105 Procurement of Medical Equipment Acquisition

106 How Effective are Financial Incentives for Attracting (and Retaining) Health Workers to Rural Areas in Uganda?

107 What are the Effective Options to Finance Private Not For Profit Health Units in Uganda?

108 Are Hospital-Based Emergency Medical Services Effective and Efficient?

109 How Can Community Health Workers be used to Empower Communities?

110 What is the Appropriate Malaria Treatment for a Low-Income Endemic Country like Uganda?

111 What Should be Included in an Optimal Package of Interventions to Prevent the Spread of HIV and Manage HIV/AIDS?

$\begin{array}{lll}\begin{array}{l}\text { Rapid } \\ \text { response } \\ \text { summary }\end{array} & \begin{array}{l}\text { Governance, coordination } \\ \text { \& M\&E }\end{array} & \begin{array}{l}\text { Governance } \\ \text { arrangements }\end{array} \\ \begin{array}{l}\text { Rapid } \\ \text { response } \\ \text { summary }\end{array} & \begin{array}{l}\text { Governance, coordination } \\ \text { \& M\&E }\end{array} & \begin{array}{l}\text { Governance } \\ \text { arrangements }\end{array} \\ \begin{array}{l}\text { Rapid } \\ \text { response } \\ \text { summary }\end{array} & \begin{array}{l}\text { Governance, coordination } \\ \text { \&apid }\end{array} & \begin{array}{l}\text { Governance } \\ \text { arrangements }\end{array} \\ \begin{array}{l}\text { response } \\ \text { summary }\end{array} & \text { \& M \&E } & \begin{array}{l}\text { Governance } \\ \text { arrangements }\end{array} \\ & & \end{array}$

Rapid

response

summary

Rapid

response

summary

Rapid

response

summary

Rapid

response

summary

Rapid

response

summary

Rapid

response

summary

Rapid

response

summary

Rapid

response

summary

Rapid

response

summary

Rapid

response

summary

Rapid

response

summary

Rapid

response

summary

Rapid

response

summary

Rapid

response

summary

Rapid

response

summary
Governance, coordination Governance

\& M\&E arrangements

Health education, Governance promotion, environmental arrangements health \& nutrition

Governance, coordination Governance \& M\&E arrangements

Health financing

Health financing

Essential medicines

\& supplies

Essential medicines \& supplies

Health financing

Health financing

Curative services

Health education, promotion, environmental arrangements health \& nutrition

Disease prevention, mitigation \& control

Disease prevention, mitigation \& control
Financial arrangements

Financial arrangements

Financial arrangements

Financial arrangements

Financial arrangements

Financial arrangements

Financial arrangements

Financial arrangements

Delivery arrangements

elivery

Delivery arrangements

Delivery arrangements
2011 SURE Project - Makerere University College of Health Sciences

2011 SURE Project - Makerere University College of Health Sciences

2011 SURE Project - Makerere University College of Health Sciences

2011 SURE Project - Makerere University College of Health Sciences

2011 SURE Project - Makerere University College of Health Sciences

2012 SURE Project - Makerere University College of Health Sciences

2013 SURE Project - Makerere University College of Health Sciences

2010 SURE Project - Makerere University College of Health Sciences

2011 SURE Project - Makerere University College of Health Sciences

2011 SURE Project - Makerere University College of Health Sciences

2011 SURE Project - Makerere University College of Health Sciences

2012 SURE Project - Makerere University College of Health Sciences

2012 SURE Project - Makerere University College of Health Sciences

2013 SURE Project - Makerere University College of Health Sciences

2013 SURE Project - Makerere University College of Health Sciences

2010 SURE Project - Makerere University College of Health Sciences

2010 SURE Project - Makerere University College of Health Sciences

2010 SURE Project - Makerere University College of Health Sciences

2010 SURE Project - Makerere University College of Health Sciences 
Table 4 Documents reviewed (Continued)

112 Dual Employment of Health Workers: Reasons and its Impact and What Steps the Gov't Can Take?

113 Health Worker Migration: What is its Impact in the Source Country? What are the Different Strategies to Implement a Bilateral Government Agreement on Recruiting Professional Health Workers from Uganda?

114 Is there an Application of Herbal Medicines (esp. D. Erecta) in the Management of HIV/AIDS and Cancer?

115 What is the Role of Stem Cell Therapy in the Management of Non-Communicable Diseases? How Does it Work and What are its Implications on the Health System?

116 Arrangement Options for Accreditation of Health Service Providers in LMICS

117 Pyrethroid Resistant Anopheles gambiae: Pyrethroid Impregnated or Synergistic LLINs?

118 What are the Effects of Clinical Pathways in Cancer Management?

119 What are the Effective Pharmaceutical Interventions for Increasing Medicines Availability in Uganda?

120 What are the Effects and Guidelines of Mass Immunization of Health Workers Against Hepatitis B?

121 How Applicable are the 2010 WHO Guidelines for Infant Feeding in the Context of HIV in LIC?

122 How Can the Sustainability of a Public Health (Food Fortification) Program be Ensured?

123 Is Mandatory Food Fortification an Efficient Strategy for the Alleviation of Micronutrient Deficiency?

124 National Policy Dialogue on the Transition and Sustainability of Public Health Nutrition Programs

125 What are the Different Strategies for Managing and Disposing of Medical Waste in Low-Income Countries?

126 What Guidelines are Present to Facilitate the Evaluation of a Natural Material Extract as a Larvicide?

127 What Health System Strategies have Low and Middle Income Countries used to Improve their Maternal Outcomes?

128 What is the Effect of Counseling in Unwanted Pregnancy?

129 What is the Evidence for the Effectiveness, Safety and Acceptability of 'Ready-To-Use Feeds'?

$\begin{array}{lll}\begin{array}{l}\text { Rapid } \\ \text { response } \\ \text { summary }\end{array} & \begin{array}{l}\text { Human resources for } \\ \text { health }\end{array} & \begin{array}{l}\text { Delivery } \\ \text { arrangements }\end{array} \\ \begin{array}{l}\text { Rapid } \\ \text { response } \\ \text { summary }\end{array} & \begin{array}{l}\text { Human resources for } \\ \text { health }\end{array} & \begin{array}{l}\text { Delivery } \\ \text { arrangements }\end{array} \\ & & \end{array}$

2011 SURE Project - Makerere University College of Health Sciences

2011 SURE Project - Makerere University College of Health Sciences

Rapid
response
summary

Rapid

response

summary

Rapid

response

summary

Rapid

response

summary

Rapid

response

summary

Rapid

response

summary

Rapid

response

summary

Rapid

response

summary

Rapid

response

summary

Rapid

response

summary

Rapid

response

summary

Rapid

response

summary

Rapid

response

summary

Rapid

response

summary

Rapid

response

summary

Rapid

response

summary
Palliative care services Delivery

arrangements

Disease prevention,

mitigation \& control

Delivery

arrangements

Human resources for health

Disease prevention, mitigation \& control

Palliative care services

supplies

Disease prevention,

mitigation \& control

Maternal and child health

Delivery

arrangements

Delivery

arrangements

Delivery

arrangements

Delivery

arrangements

Implementation

strategies

Implementation strategies

Health education, Implementation 2011 SURE Project - Makerere promotion, environmental strategies health \& nutrition

Health education, Health Sciences

2011 SURE Project - Makerere promotion, environmental strategies University College of health \& nutrition Health Sciences

Health education, Implementation 2011

promotion, environmental strategies health \& nutrition

SURE Project - Makerere

Health education, Implementation 2011 SURE Project - Makerere promotion, environmental strategies University College of health \& nutrition Health Sciences

Disease prevention, mitigation \& control

Implementation 2011

SURE Project - Makerere University College of Health Sciences

Maternal and child health Implementation 2011 SURE Project - Makerere strategies University College of Health Sciences

Reproductive health

Implementation 2011 SURE Project - Makerere strategies University College of Health Sciences

Health education, Implementation 2011 SURE Project - Makerere promotion, environmental strategies health \& nutrition
University College of Health Sciences 
Table 4 Documents reviewed (Continued)

130 What Strategies can Health Systems in Low-Income Settings Employ for Infection Control?

131 Research to Policy at the NTLP Uganda

32 What are the Health Effects (Benefits and Risks) of Steam Baths (Saunas)?

133 Task Shifting for Health Workers in Maternal and Child Healthcare

134 Improving Access to Skilled Attendance at Delivery

135 National Framework for Sustainability of Health Knowledge Translation Initiatives in Uganda

136 Mainstreaming Nutrition with Agriculture in Uganda: Role of Agriculture in Improving the Nutritional Status of Women and Children

137 Establishing the Advisory Committee on Vaccines and Immunization

138 Preventing a Tobacco Epidemic in Africa. A Call For Effective Action to Support Health, Social, and Economic Development

139 The Role of Science Academies in Generating Evidence-Based Advice for Effective Policy Decision Making: The Case of Climate Change (Hosted By The Uganda National Academy Of Sciences (UNAS), Hotel African, Kampala-Uganda, 11th-12th October, 2010)

140 Policy and Strategy for Insecticide Treated Nets

141 National Policy on HIV/AIDS and the World of Work

142 The National Youth Policy

143 National Orphans and Other Vulnerable Children Policy

144 The Occupational Safety and Health Act, 2007

145 Antiretroviral Treatment Policy for Uganda

146 National Policy on Adolescent Health

147 National Environmental Health Policy

148 National Oral Health Policy

149 National Policy Public Private Partnership in Health

150 Uganda National Drug Policy

\begin{tabular}{|c|c|c|c|c|}
\hline $\begin{array}{l}\text { Rapid } \\
\text { response } \\
\text { summary }\end{array}$ & $\begin{array}{l}\text { Disease prevention, } \\
\text { mitigation \& control }\end{array}$ & $\begin{array}{l}\text { Implementation } \\
\text { strategies }\end{array}$ & 2012 & $\begin{array}{l}\text { SURE Project - Makerere } \\
\text { University College of } \\
\text { Health Sciences }\end{array}$ \\
\hline $\begin{array}{l}\text { Rapid } \\
\text { response } \\
\text { summary }\end{array}$ & Others & Other & 2012 & $\begin{array}{l}\text { SURE Project - Makerere } \\
\text { University College of } \\
\text { Health Sciences }\end{array}$ \\
\hline $\begin{array}{l}\text { Rapid } \\
\text { response } \\
\text { summary }\end{array}$ & $\begin{array}{l}\text { Governance, coordination } \\
\& \text { M\&E }\end{array}$ & Other & 2012 & $\begin{array}{l}\text { SURE Project - Makerere } \\
\text { University College of } \\
\text { Health Sciences }\end{array}$ \\
\hline $\begin{array}{l}\text { Policy } \\
\text { dialogue } \\
\text { report }\end{array}$ & $\begin{array}{l}\text { Human resources for } \\
\text { health }\end{array}$ & $\begin{array}{l}\text { Delivery } \\
\text { arrangements }\end{array}$ & 2010 & $\begin{array}{l}\text { SURE Project - Makerere } \\
\text { University College of } \\
\text { Health Sciences }\end{array}$ \\
\hline $\begin{array}{l}\text { Policy } \\
\text { dialogue } \\
\text { report }\end{array}$ & Maternal and child health & $\begin{array}{l}\text { Delivery } \\
\text { arrangements }\end{array}$ & 2011 & $\begin{array}{l}\text { SURE Project - Makerere } \\
\text { University College of } \\
\text { Health Sciences }\end{array}$ \\
\hline $\begin{array}{l}\text { Policy } \\
\text { dialogue } \\
\text { report }\end{array}$ & Others & Other & 2014 & $\begin{array}{l}\text { SURE Project - Makerere } \\
\text { University College of } \\
\text { Health Sciences }\end{array}$ \\
\hline $\begin{array}{l}\text { Policy } \\
\text { dialogue } \\
\text { report }\end{array}$ & $\begin{array}{l}\text { Health education, } \\
\text { promotion, environmental } \\
\text { health \& nutrition }\end{array}$ & $\begin{array}{l}\text { Governance } \\
\text { arrangements }\end{array}$ & 2011 & $\begin{array}{l}\text { Uganda National Academy } \\
\text { of Sciences }\end{array}$ \\
\hline $\begin{array}{l}\text { Policy } \\
\text { dialogue } \\
\text { report }\end{array}$ & $\begin{array}{l}\text { Disease prevention, } \\
\text { mitigation \& control }\end{array}$ & $\begin{array}{l}\text { Governance } \\
\text { arrangements }\end{array}$ & 2012 & $\begin{array}{l}\text { Uganda National Academy } \\
\text { of Sciences }\end{array}$ \\
\hline $\begin{array}{l}\text { Policy } \\
\text { dialogue } \\
\text { report }\end{array}$ & $\begin{array}{l}\text { Disease prevention, } \\
\text { mitigation \& control }\end{array}$ & $\begin{array}{l}\text { Implementation } \\
\text { strategies }\end{array}$ & 2011 & $\begin{array}{l}\text { Uganda National Academy } \\
\text { of Sciences }\end{array}$ \\
\hline $\begin{array}{l}\text { licy } \\
\text { alogue }\end{array}$ & Others & Other & 2010 & $\begin{array}{l}\text { Uganda National Academy } \\
\text { of Sciences }\end{array}$ \\
\hline
\end{tabular}

\begin{tabular}{|c|c|c|c|c|}
\hline Policy & $\begin{array}{l}\text { Disease prevention, } \\
\text { mitigation \& control }\end{array}$ & $\begin{array}{l}\text { Governance } \\
\text { arrangements }\end{array}$ & 2003 & Malaria Control Programme \\
\hline Policy & $\begin{array}{l}\text { Disease prevention, } \\
\text { mitigation \& control }\end{array}$ & $\begin{array}{l}\text { Governance } \\
\text { arrangements }\end{array}$ & 2007 & $\begin{array}{l}\text { Ministry of Gender, Labour } \\
\text { and Social Development }\end{array}$ \\
\hline Policy & Others & $\begin{array}{l}\text { Governance } \\
\text { arrangements }\end{array}$ & 2001 & $\begin{array}{l}\text { Ministry of Gender, Labour } \\
\text { and Social Development }\end{array}$ \\
\hline Policy & Others & $\begin{array}{l}\text { Governance } \\
\text { arrangements }\end{array}$ & 2004 & $\begin{array}{l}\text { Ministry of Gender, Labour } \\
\text { and Social Development }\end{array}$ \\
\hline Policy & $\begin{array}{l}\text { Disease prevention, } \\
\text { mitigation \& control }\end{array}$ & Other & 2007 & Ministry of Health \\
\hline Policy & $\begin{array}{l}\text { Disease prevention, } \\
\text { mitigation \& control }\end{array}$ & $\begin{array}{l}\text { Governance } \\
\text { arrangements }\end{array}$ & 2003 & Ministry of Health \\
\hline Policy & $\begin{array}{l}\text { Health education, } \\
\text { promotion, environmental } \\
\text { health \& nutrition }\end{array}$ & $\begin{array}{l}\text { Governance } \\
\text { arrangements }\end{array}$ & 2004 & Ministry of Health \\
\hline Policy & $\begin{array}{l}\text { Health education, } \\
\text { promotion, environmental } \\
\text { health \& nutrition }\end{array}$ & $\begin{array}{l}\text { Governance } \\
\text { arrangements }\end{array}$ & 2005 & Ministry of Health \\
\hline Policy & $\begin{array}{l}\text { Disease prevention, } \\
\text { mitigation \& control }\end{array}$ & $\begin{array}{l}\text { Governance } \\
\text { arrangements }\end{array}$ & 2007 & Ministry of Health \\
\hline Policy & Others & $\begin{array}{l}\text { Governance } \\
\text { arrangements }\end{array}$ & 2012 & Ministry of Health \\
\hline Policy & $\begin{array}{l}\text { Essential medicines \& } \\
\text { supplies }\end{array}$ & Other & 2002 & Ministry of Health \\
\hline
\end{tabular}


Table 4 Documents reviewed (Continued)

\begin{tabular}{|c|c|c|c|c|c|c|}
\hline 151 & National Anaemia Policy & Policy & $\begin{array}{l}\text { Health education, } \\
\text { promotion, environmental } \\
\text { health \& nutrition }\end{array}$ & Other & 2002 & Ministry of Health \\
\hline 152 & National Hospital Policy & Policy & Curative services & Other & 2004 & Ministry of Health \\
\hline 153 & National Policy on Malaria Treatment & Policy & $\begin{array}{l}\text { Disease prevention, } \\
\text { mitigation \& control }\end{array}$ & Other & 2005 & Ministry of Health \\
\hline 154 & $\begin{array}{l}\text { National Policy on Public Health } \\
\text { Sector Monitoring and Evaluation (M\&E) }\end{array}$ & Policy & $\begin{array}{l}\text { Governance, coordination } \\
\& M \& E\end{array}$ & $\begin{array}{l}\text { Governance } \\
\text { arrangements }\end{array}$ & 2013 & $\begin{array}{l}\text { Office of the Prime } \\
\text { Minister }\end{array}$ \\
\hline 155 & $\begin{array}{l}\text { Animal Diseases (Selective Importation } \\
\text { of Livestock, Livestock Products, } \\
\text { Co-Products and By-Products) } \\
\text { Regulations, } 2003\end{array}$ & Policy & $\begin{array}{l}\text { Disease prevention, } \\
\text { mitigation \& control }\end{array}$ & Other & 2003 & Parliament of Uganda \\
\hline 156 & $\begin{array}{l}\text { Electricity (Safety Code) Regulations, } \\
2003\end{array}$ & Policy & $\begin{array}{l}\text { Disease prevention, } \\
\text { mitigation \& control }\end{array}$ & Other & 2003 & Parliament of Uganda \\
\hline 157 & $\begin{array}{l}\text { The National Environment (Control of } \\
\text { Smoking in Public Places) Regulations, } \\
2004\end{array}$ & Policy & $\begin{array}{l}\text { Disease prevention, } \\
\text { mitigation \& control }\end{array}$ & Other & 2004 & Parliament of Uganda \\
\hline 158 & $\begin{array}{l}\text { Births and Deaths Registration } \\
\text { (Amendment) Regulations, } 2005\end{array}$ & Policy & Others & Other & 2005 & Parliament of Uganda \\
\hline 159 & $\begin{array}{l}\text { Agricultural Chemicals (Control) } \\
\text { Act, } 2006\end{array}$ & Policy & $\begin{array}{l}\text { Health education, } \\
\text { promotion, environmental } \\
\text { health \& nutrition }\end{array}$ & Other & 2006 & Parliament of Uganda \\
\hline 160 & $\begin{array}{l}\text { HIV and AIDS Prevention and } \\
\text { Control Act, } 2014\end{array}$ & Policy & $\begin{array}{l}\text { Disease prevention, } \\
\text { mitigation \& control }\end{array}$ & Other & 2014 & Parliament of Uganda \\
\hline 161 & $\begin{array}{l}\text { Population Policy for Social } \\
\text { Transformation and Sustainable } \\
\text { Development }\end{array}$ & Policy & Others & Other & 2009 & Population Secretariat \\
\hline 162 & $\begin{array}{l}\text { The Uganda Food and Nutrition } \\
\text { Policy }\end{array}$ & Policy & $\begin{array}{l}\text { Health education, } \\
\text { promotion, environmental } \\
\text { health \& nutrition }\end{array}$ & Other & 2003 & $\begin{array}{l}\text { SURE Project - Makerere } \\
\text { University College of } \\
\text { Health Sciences }\end{array}$ \\
\hline 163 & $\begin{array}{l}\text { Uganda HIV Counseling and Testing } \\
\text { 3rd Policy Edition }\end{array}$ & Policy & $\begin{array}{l}\text { Disease prevention, } \\
\text { mitigation \& control }\end{array}$ & Other & 2010 & Uganda AIDS Commission \\
\hline 164 & Safe Male Circumcision Policy & Policy & $\begin{array}{l}\text { Disease prevention, } \\
\text { mitigation \& control }\end{array}$ & $\begin{array}{l}\text { Governance } \\
\text { arrangements }\end{array}$ & 2010 & $\begin{array}{l}\text { Uganda Bureau of } \\
\text { Statistics }\end{array}$ \\
\hline 165 & $\begin{array}{l}\text { Uganda National Health Laboratory } \\
\text { Service Policy }\end{array}$ & Policy & Health infrastructure & $\begin{array}{l}\text { Governance } \\
\text { arrangements }\end{array}$ & 2009 & $\begin{array}{l}\text { Uganda Community Based } \\
\text { Health Financing Association }\end{array}$ \\
\hline 166 & Uganda National HIV/AIDS Policy & Policy & $\begin{array}{l}\text { Disease prevention, } \\
\text { mitigation \& control }\end{array}$ & Other & 2011 & $\begin{array}{l}\text { Uganda Community Based } \\
\text { Health Financing Association }\end{array}$ \\
\hline 167 & $\begin{array}{l}\text { National Biotechnology and Biosafety } \\
\text { Policy, } 2008\end{array}$ & Policy & $\begin{array}{l}\text { Disease prevention, } \\
\text { mitigation \& control }\end{array}$ & $\begin{array}{l}\text { Governance } \\
\text { arrangements }\end{array}$ & 2008 & $\begin{array}{l}\text { Uganda National Council } \\
\text { for Science and Technology }\end{array}$ \\
\hline 168 & $\begin{array}{l}\text { National Science, Technology and } \\
\text { Innovation Policy, } 2009\end{array}$ & Policy & Others & $\begin{array}{l}\text { Governance } \\
\text { arrangements }\end{array}$ & 2009 & $\begin{array}{l}\text { Uganda National Council } \\
\text { for Science and Technology }\end{array}$ \\
\hline 169 & National Population Policy 2008 & Policy & Others & Other & 2008 & $\begin{array}{l}\text { Uganda Population } \\
\text { Secretariat }\end{array}$ \\
\hline 170 & National Policy on Disability & Policy & Rehabilitation services & $\begin{array}{l}\text { Governance } \\
\text { arrangements }\end{array}$ & 2006 & World Health Organization \\
\hline 171 & $\begin{array}{l}\text { National Policy on Traditional Medicine } \\
\text { and Regulation of Herbal Medicines }\end{array}$ & Policy & $\begin{array}{l}\text { Essential medicines \& } \\
\text { supplies }\end{array}$ & Other & 2005 & World Health Organization \\
\hline 172 & $\begin{array}{l}\text { Policy for the Reduction of the Mother-to- } \\
\text { Child HIV Transmission in Uganda (2003) }\end{array}$ & Policy & $\begin{array}{l}\text { Disease prevention, } \\
\text { mitigation \& control }\end{array}$ & $\begin{array}{l}\text { Governance } \\
\text { arrangements }\end{array}$ & 2003 & Ministry of Health \\
\hline 173 & $\begin{array}{l}\text { Uganda Food And Nutrition Strategy } \\
\text { And Investment Plan }\end{array}$ & Plan & $\begin{array}{l}\text { Health education, } \\
\text { promotion, environmental } \\
\text { health \& nutrition }\end{array}$ & $\begin{array}{l}\text { Implementation } \\
\text { strategies }\end{array}$ & 2004 & Ministry of Agriculture \\
\hline 174 & National Action Plan & Plan & $\begin{array}{l}\text { Governance, coordination } \\
\& \text { M\&E }\end{array}$ & $\begin{array}{l}\text { Implementation } \\
\text { strategies }\end{array}$ & 2011 & $\begin{array}{l}\text { Ministry of Gender, Labour } \\
\text { and Social Development }\end{array}$ \\
\hline
\end{tabular}


Table 4 Documents reviewed (Continued)

175 National Action Plan on Elimination of the Worst Forms of Child Labour in Uganda

176 Uganda Gender Action Plan

177 M\&E for HSSIP 2010/11-2014/2015

178 National Malaria Action Plan

179 Roadmap for Accelerating the Reduction of Maternal and Neonatal Mortality and Morbidity

180 Nutrition HIV Communication Strategy

181 Nutrition in the Context of HIV and TB

182 Health Sector Strategic and Investment Plan

183 Health Sector Quality Improvement Strategic Plan \& Framework

184 Uganda Nutrition Action Plan

185 Uganda Malaria Control Strategic Plan 2005/06

186 National Development Plan

187 Uganda Vision 2040

188 Pharmaceutical Society of Uganda Strategic Plan

189 The National Population Policy Action Plan

190 National HIV \& AIDS M\&E Plan 2011/2-2014/15

191 National Priority Action Plan

192 Uganda National Expanded Programme on Immunization Multi Year Plan 2010-2014

193 National Science, Technology and Innovation Plan 2012/2013-2017/2018

194 UNEPI on Immunization Multi-Year Plan 2012-2016

195 The Nation Population Policy Action Plan 2011-2015

196 The Uganda National Plan of Action on Child Sexual Abuse and Exploitation

197 Malaria Operational Plan FY 2015

198 Guidelines on Recruitment of Health Workers in Districts and Urban Authorities
Plan

Plan

Plan

Plan

Plan

Plan

Plan

Plan

Plan

Plan

Plan

Plan

Plan

Plan

Plan

Plan

Plan

Plan

Plan

Plan

Plan

Plan

Plan

Guidelines
Maternal and child health

strategies

Ministry of Gender, Labour and Social Development

Others

Implementation 2010 Ministry of Gender, Labour strategies and Social Development

Governance, coordination Delivery 2011 Ministry of Health \& M\&E

arrangements

Disease prevention, mitigation \& control

Implementation 2006 Ministry of Health strategies

Maternal and child health Implementation 2007 Ministry of Health strategies

Health education, Implementation 2009 Ministry of Health promotion, environmental strategies

health \& nutrition

Health education, Implementation 2009 Ministry of Health promotion, environmental strategies

health \& nutrition

Governance, coordination Implementation 2010 Ministry of Health \& M\&E strategies

Governance, coordination Implementation 2011 Ministry of Health \& M\&E strategies

Health education, Implementation 2011 Ministry of Health promotion, environmental strategies

health \& nutrition

Disease prevention, Implementation 2005 National Malaria Control mitigation \& control strategies Programme

Governance, coordination Implementation 2010 National Planning Authority \& M\&E strategies

Governance, coordination Implementation 2013 National Planning Authority \& M\&E strategies

Essential medicines \& Implementation 2013 Pharmaceutical Society supplies

strategies of Uganda

Governance, coordination Implementation 2011 Population Secretariat \& M\&E strategies

Governance, coordination Implementation 2011 Uganda AIDS Commission \& M\&E

strategies

Disease prevention, Implementation 2011 Uganda AIDS Commission mitigation \& control strategies

Health education, Implementation 2010 Uganda Expanded

promotion, environmental strategies Programme on Immunisation health \& nutrition

Others

Implementation 2012 Uganda National Council strategies for Science and Technology

Disease prevention, mitigation \& control

Implementation 2012 Uganda National Expanded strategies Programme on Immunisation

Governance, coordination Implementation 2011 Uganda Population $\& M \& E$

strategies Secretariat

Maternal and child health Implementation 2008 UNICEF strategies

Disease prevention, mitigation \& control

Implementation 2014 USAID strategies

Human resources for health
Implementation 2005 Health Service Commission strategies 
Table 4 Documents reviewed (Continued)

199 The Integrated National Guidelines on Antiretroviral Therapy Prevention of Mother to Child Transmission of HIV Infant \& Young Child Feeding

200 Education \& Sports Sector National Policy Guidelines on HIV/AIDS

201 Guidelines on Hospital Management Committees for District Hospitals

202 Uganda National Guidelines and Service Standard for Reproductive Health Services

203 Guidelines on Health Unit Management Committees for Health Centre 3

204 Health Planning Guidelines - Supplement to the Local Government Planning Process

205 National Guidelines

206 Patients' Charter

207 National Clinical Guidelines

208 Policy Guidelines and Service Delivery Standards for Community Based Provision of Injectable Contraception in Uganda

209 Guidelines for Designation, Establishment and Upgrading of Health Units

210 Implementation Guidelines for Integrated Community Case Management of Childhood Malaria, Pneumonia and Diarrhea

211 Guidelines on Nutrition Survey Methodology in Uganda

212 Nutritional Care and Support for People Living with HIV/AIDS in Uganda: Guidelines for Service Providers

213 National Guidelines for Establishment and Scaling Up of VHTs

214 Guidelines for Establishing and Operating Couple's Clubs

215 National Guidelines

216 Feeding Guidelines for People Living with HIV/AIDS: A Handbook for Field Extension Workers

217 Guide to Ideal Feeding Practices: For People with Increased Nutritional Needs, Care and Support

218 The Local Government Development Planning Guidelines

219 Guidelines for Breast cancer

220 National Guidelines
Guidelines

Disease prevention, mitigation \& control

Governance

arrangements

2012 Infectious Diseases Institute

\section{Guidelines}

Disease prevention, mitigation \& control

Implementation 2006 Ministry of Education strategies

Guidelines

Governance, coordination $\& M \& E$

Governance

arrangements

Guidelines

Reproductive health

Governance arrangements

Guidelines

Governance, coordination $\& M \& E$

Governance arrangements

Guidelines

Governance, coordination $\& M \& E$

Governance arrangements

Guidelines

Health education,

Governance promotion, environmental arrangements health \& nutrition

Guidelines

Governance, coordination \& M\&E

Governance arrangements

Guidelines

Disease prevention, mitigation \& control

Governance arrangements

Guidelines

Reproductive health

Governance arrangements

Guidelines

Guidelines

Health infrastructure

Governance arrangements

Maternal and child health Delivery arrangements

Guidelines

Health education,

Delivery promotion, environmental arrangements health \& nutrition

Guidelines

Health education,

Delivery promotion, environmental arrangements health \& nutrition

Guidelines

Health education, Implementation 2009 Ministry of Health promotion, environmental strategies health \& nutrition

Guidelines

Health education, Implementation 2011 Ministry of Health promotion, environmental strategies health \& nutrition

Guidelines

Health education, Implementation promotion, environmental strategies health \& nutrition

Guidelines

Human resources for health

Guidelines

Health education, promotion, environmenta health \& nutrition

Guidelines

Governance, coordination \& M\&E arrangements

Guidelines

Disease prevention, mitigation \& control

Guidelines
Governance

Governance arrangements

Governance arrangements

Other

Governance arrangements
2000 Ministry of Health

2001 Ministry of Health

2003 Ministry of Health

2007 Ministry of Health

2008 Ministry of Health

2009 Ministry of Health

2010 Ministry of Health

2010 Ministry of Health

2011 Ministry of Health

2000 Ministry of Health

2009 Ministry of Health

2012 Ministry of Health
Disease prevention, mitigation \& control 
Table 4 Documents reviewed (Continued)

221 National Guidelines for Maternal and Child Health

222 National Implementation Guidelines for HIV Counseling and Testing in Uganda

223 Uganda Guidelines for AIDS Vaccine Research. A Guide for Vaccine Research, Development and Evaluation

224 Uganda National Expended Programme on Immunisation Standards

225 Guidelines in Respect of Complaints against Medical and Dental Practitioners

226 Guidelines and Standards for Accreditation of Continuing Professional Development for Health Workers

227 National Guidelines on Bio-ethics

228 National Guidelines for Field Trials of Genetically Engineered Plants

229 Guidelines for Accreditation of Research Ethics Committees

230 Treatment of Tuberculosis: Guidelines for National Programmes - 3rd Edition

231 Guidelines on Malaria Treatment

232 Policy Guidelines on Feeding of Infants and Young Children in the Context of HIV/AIDS (2001)

233 Battling Water-Borne Disease amongst Children: An Assessment of Policy Option from Uganda

234 Medicines for Life: Clients and Providers in Uganda

235 Financing of Reproductive Health Services in Uganda

236 Eliminating Congenital Syphilis in Uganda

237 Gender and Health Policy Brief for Uganda

238 Youth Reproductive Health Policy

239 Global Fund: Making Uganda's CCM Work Through Full Engagement of Civil Society

240 The Industrial Property Bill 2007 Balancing Inventors Rights with Public Health Interests in Uganda's IP Legislation

241 Making UNSCR 1325, 1820 and the Goma Declaration a Reality for Women and Girls in Uganda

242 CSOs Position on National Pharmaceutical Plan

\begin{tabular}{|c|c|c|c|c|}
\hline Guidelines & Maternal and child health & $\begin{array}{l}\text { Governance } \\
\text { arrangements }\end{array}$ & 2010 & $\begin{array}{l}\text { SURE Project - Makerere } \\
\text { University College of } \\
\text { Health Sciences }\end{array}$ \\
\hline Guidelines & $\begin{array}{l}\text { Disease prevention, } \\
\text { mitigation \& control }\end{array}$ & $\begin{array}{l}\text { Governance } \\
\text { arrangements }\end{array}$ & 2010 & Uganda AIDS Commission \\
\hline Guidelines & $\begin{array}{l}\text { Disease prevention, } \\
\text { mitigation \& control }\end{array}$ & Other & 2000 & Uganda AIDS Commission \\
\hline Guidelines & $\begin{array}{l}\text { Disease prevention, } \\
\text { mitigation \& control }\end{array}$ & $\begin{array}{l}\text { Governance } \\
\text { arrangements }\end{array}$ & 2003 & $\begin{array}{l}\text { Uganda Expanded } \\
\text { Programme on Immunisation }\end{array}$ \\
\hline Guidelines & $\begin{array}{l}\text { Governance, coordination } \\
\& \text { M\&E }\end{array}$ & $\begin{array}{l}\text { Governance } \\
\text { arrangements }\end{array}$ & 2002 & $\begin{array}{l}\text { Uganda Medical Practitioners } \\
\text { Council }\end{array}$ \\
\hline Guidelines & $\begin{array}{l}\text { Governance, coordination } \\
\& M \& E\end{array}$ & $\begin{array}{l}\text { Governance } \\
\text { arrangements }\end{array}$ & 2008 & $\begin{array}{l}\text { Uganda Medical Practitioners } \\
\text { Council }\end{array}$ \\
\hline Guidelines & $\begin{array}{l}\text { Governance, coordination } \\
\& \text { M\&E }\end{array}$ & $\begin{array}{l}\text { Governance } \\
\text { arrangements }\end{array}$ & 2007 & $\begin{array}{l}\text { Uganda National Council } \\
\text { for Science and Technology }\end{array}$ \\
\hline Guidelines & $\begin{array}{l}\text { Governance, coordination } \\
\& M \& E\end{array}$ & $\begin{array}{l}\text { Governance } \\
\text { arrangements }\end{array}$ & 2011 & $\begin{array}{l}\text { Uganda National Council } \\
\text { for Science and Technology }\end{array}$ \\
\hline Guidelines & $\begin{array}{l}\text { Governance, coordination } \\
\& M \& E\end{array}$ & $\begin{array}{l}\text { Governance } \\
\text { arrangements }\end{array}$ & 2014 & $\begin{array}{l}\text { Uganda National Council } \\
\text { for Science and Technology }\end{array}$ \\
\hline Guidelines & $\begin{array}{l}\text { Disease prevention, } \\
\text { mitigation \& control }\end{array}$ & $\begin{array}{l}\text { Delivery } \\
\text { arrangements }\end{array}$ & 2003 & World Health Organization \\
\hline Guidelines & $\begin{array}{l}\text { Disease prevention, } \\
\text { mitigation \& control }\end{array}$ & $\begin{array}{l}\text { Delivery } \\
\text { arrangements }\end{array}$ & 2010 & World Health Organization \\
\hline Guidelines & $\begin{array}{l}\text { Disease prevention, } \\
\text { mitigation \& control }\end{array}$ & Other & 2001 & Ministry of Health \\
\hline $\begin{array}{l}\text { Evidence } \\
\text { brief for } \\
\text { policy }\end{array}$ & $\begin{array}{l}\text { Health education, } \\
\text { promotion, environmental } \\
\text { health \& nutrition }\end{array}$ & $\begin{array}{l}\text { Delivery } \\
\text { arrangements }\end{array}$ & 2010 & $\begin{array}{l}\text { Centre for Environmental } \\
\text { Economics and Policy in } \\
\text { Africa (CEEPA) }\end{array}$ \\
\hline $\begin{array}{l}\text { Evidence } \\
\text { brief for } \\
\text { policy }\end{array}$ & $\begin{array}{l}\text { Essential medicines \& } \\
\text { supplies }\end{array}$ & $\begin{array}{l}\text { Delivery } \\
\text { arrangements }\end{array}$ & 2014 & $\begin{array}{l}\text { Child Health and } \\
\text { Development Centre }\end{array}$ \\
\hline $\begin{array}{l}\text { Evidence } \\
\text { brief for } \\
\text { policy }\end{array}$ & Health financing & $\begin{array}{l}\text { Financial } \\
\text { arrangements }\end{array}$ & 2012 & $\begin{array}{l}\text { Ahead for World Bank } \\
\text { Advocacy Coalition }\end{array}$ \\
\hline $\begin{array}{l}\text { Evidence } \\
\text { brief for } \\
\text { policy }\end{array}$ & $\begin{array}{l}\text { Disease prevention, } \\
\text { mitigation \& control }\end{array}$ & $\begin{array}{l}\text { Delivery } \\
\text { arrangements }\end{array}$ & 2011 & $\begin{array}{l}\text { Elizabeth Glaser Pediatric } \\
\text { AIDS Foundation }\end{array}$ \\
\hline $\begin{array}{l}\text { Evidence } \\
\text { brief for } \\
\text { policy }\end{array}$ & Others & $\begin{array}{l}\text { Governance } \\
\text { arrangements }\end{array}$ & 2014 & $\begin{array}{l}\text { Forum for Women in } \\
\text { Democracy }\end{array}$ \\
\hline $\begin{array}{l}\text { Evidence } \\
\text { brief for } \\
\text { policy }\end{array}$ & Reproductive health & $\begin{array}{l}\text { Governance } \\
\text { arrangements }\end{array}$ & 2005 & HEPS Uganda \\
\hline $\begin{array}{l}\text { Evidence } \\
\text { brief for } \\
\text { policy }\end{array}$ & $\begin{array}{l}\text { Governance, coordination } \\
\& \text { M\&E }\end{array}$ & $\begin{array}{l}\text { Governance } \\
\text { arrangements }\end{array}$ & 2008 & HEPS Uganda \\
\hline $\begin{array}{l}\text { Evidence } \\
\text { brief for } \\
\text { policy }\end{array}$ & $\begin{array}{l}\text { Governance, coordination } \\
\& \text { M\&E }\end{array}$ & $\begin{array}{l}\text { Governance } \\
\text { arrangements }\end{array}$ & 2008 & HEPS Uganda \\
\hline $\begin{array}{l}\text { Evidence } \\
\text { brief for } \\
\text { policy }\end{array}$ & Maternal and child health & $\begin{array}{l}\text { Governance } \\
\text { arrangements }\end{array}$ & 2010 & HEPS Uganda \\
\hline $\begin{array}{l}\text { Evidence } \\
\text { brief for }\end{array}$ & $\begin{array}{l}\text { Governance, coordination } \\
\& \text { M\&E }\end{array}$ & $\begin{array}{l}\text { Governance } \\
\text { arrangements }\end{array}$ & 2014 & HEPS Uganda \\
\hline
\end{tabular}

Guidelines Maternal and child health Governance

Disease prevention,

Disease prevention, arrangements

ther

Governance

Governance

rangements

Governance

Governance

Governance, coordination Governance

Delivery arrangements arrangements

Disease prevention,

Health education, Delivery health \& nutrition

Essential medicines $\&$

Delivery

policy

policy

vidence

Evidence

Evidence

brief for

policy

brief for

policy

policy

Evidence

brief for

Evidence

policy
\& M\&E

arrangements

University College of

000 Uganda AIDS Commission

Uganda Expanded

Programme on Immunisation

008 Uganda Medical Practitioners

.

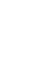


Table 4 Documents reviewed (Continued)

243 Improving the Availability and Management of Essential AIDS and TB Medicines and Diagnostics in Uganda

244 A National Framework for Sustainability of Health Knowledge Translation Initiatives in Uganda

245 Community Involvement in HIV Prevention Research: Successes and Failures

246 National Strategy for Girls' Education in Uganda

247 Gaps in the Implementation of Reproductive Health Policies in Uganda

248 Sustainable Coverage of LLINs in Uganda

249 Mental Health Law Reforms in Uganda

250 Integration of Mental Health into Primary Healthcare in Uganda: Success and Challenges

251 Community Case Management of Malaria

252 Adolescent Sexual and Reproductive Health and Rights in the Post-2015 Agenda

253 Child Protection in the Post-2015 Agenda

254 Advancing the Integration of Palliative Care in the National Health System

255 Policy Brief on Improving Access to Artemisinin-Based Combination Therapies for Malaria in the East African Community

256 Task Shifting to Optimize the Roles of Health Workers to Improve the Delivery of Maternal and Child Healthcare

257 Improving Access to Skilled Attendance at Delivery

258 From Commitment to Action: The RAPID Application in Uganda

259 Integrating Nutrition and Agriculture: Use of Extension Workers and Community Models in Uganda

260 Improving Vaccine and Immunization Coverage in Uganda

261 Observing our Commitment to Addressing Gender Based Violence and Reproductive Rights in Uganda

\begin{tabular}{|c|c|c|c|c|}
\hline $\begin{array}{l}\text { Evidence } \\
\text { brief for } \\
\text { policy }\end{array}$ & $\begin{array}{l}\text { Essential medicines \& } \\
\text { supplies }\end{array}$ & $\begin{array}{l}\text { Delivery } \\
\text { arrangements }\end{array}$ & 2008 & HEPS Uganda \\
\hline $\begin{array}{l}\text { Evidence } \\
\text { brief for } \\
\text { policy }\end{array}$ & Others & $\begin{array}{l}\text { Delivery } \\
\text { arrangements }\end{array}$ & 2014 & HEPS Uganda \\
\hline $\begin{array}{l}\text { Evidence } \\
\text { brief for } \\
\text { policy }\end{array}$ & $\begin{array}{l}\text { Disease prevention, } \\
\text { mitigation \& control }\end{array}$ & $\begin{array}{l}\text { Implementation } \\
\text { strategies }\end{array}$ & 2010 & HEPS Uganda \\
\hline $\begin{array}{l}\text { Evidence } \\
\text { brief for } \\
\text { policy }\end{array}$ & Maternal and child health & $\begin{array}{l}\text { Implementation } \\
\text { strategies }\end{array}$ & 2014 & HEPS Uganda \\
\hline $\begin{array}{l}\text { Evidence } \\
\text { brief for } \\
\text { policy }\end{array}$ & Reproductive health & $\begin{array}{l}\text { Implementation } \\
\text { strategies }\end{array}$ & 2012 & Isis-WICCE \\
\hline $\begin{array}{l}\text { Evidence } \\
\text { brief for } \\
\text { policy }\end{array}$ & $\begin{array}{l}\text { Disease prevention, } \\
\text { mitigation \& control }\end{array}$ & $\begin{array}{l}\text { Implementation } \\
\text { strategies }\end{array}$ & 2014 & Malaria Consortium \\
\hline $\begin{array}{l}\text { Evidence } \\
\text { brief for } \\
\text { policy }\end{array}$ & $\begin{array}{l}\text { Governance, coordination } \\
\& \text { M\&E }\end{array}$ & $\begin{array}{l}\text { Governance } \\
\text { arrangements }\end{array}$ & 2014 & $\begin{array}{l}\text { Mental Health and } \\
\text { Poverty Project }\end{array}$ \\
\hline $\begin{array}{l}\text { Evidence } \\
\text { brief for } \\
\text { policy }\end{array}$ & Curative services & $\begin{array}{l}\text { Delivery } \\
\text { arrangements }\end{array}$ & 2014 & $\begin{array}{l}\text { Mental Health and } \\
\text { Poverty Project }\end{array}$ \\
\hline $\begin{array}{l}\text { Evidence } \\
\text { brief for } \\
\text { policy }\end{array}$ & $\begin{array}{l}\text { Disease prevention, } \\
\text { mitigation \& control }\end{array}$ & $\begin{array}{l}\text { Delivery } \\
\text { arrangements }\end{array}$ & 2014 & Ministry of Health \\
\hline $\begin{array}{l}\text { Evidence } \\
\text { brief for } \\
\text { policy }\end{array}$ & Reproductive health & $\begin{array}{l}\text { Governance } \\
\text { arrangements }\end{array}$ & 2014 & Plan International \\
\hline $\begin{array}{l}\text { Evidence } \\
\text { brief for } \\
\text { policy }\end{array}$ & Others & $\begin{array}{l}\text { Governance } \\
\text { arrangements }\end{array}$ & 2014 & Plan International \\
\hline $\begin{array}{l}\text { Evidence } \\
\text { brief for } \\
\text { policy }\end{array}$ & Palliative care services & $\begin{array}{l}\text { Governance } \\
\text { arrangements }\end{array}$ & 2013 & $\begin{array}{l}\text { SURE Project - Makerere } \\
\text { University College of } \\
\text { Health Sciences }\end{array}$ \\
\hline $\begin{array}{l}\text { Evidence } \\
\text { brief for } \\
\text { policy }\end{array}$ & $\begin{array}{l}\text { Essential medicines \& } \\
\text { supplies }\end{array}$ & $\begin{array}{l}\text { Delivery } \\
\text { arrangements }\end{array}$ & 2010 & $\begin{array}{l}\text { SURE Project - Makerere } \\
\text { University College of } \\
\text { Health Sciences }\end{array}$ \\
\hline $\begin{array}{l}\text { Evidence } \\
\text { brief for } \\
\text { policy }\end{array}$ & $\begin{array}{l}\text { Human resources for } \\
\text { health }\end{array}$ & $\begin{array}{l}\text { Delivery } \\
\text { arrangements }\end{array}$ & 2010 & $\begin{array}{l}\text { SURE Project - Makerere } \\
\text { University College of } \\
\text { Health Sciences }\end{array}$ \\
\hline $\begin{array}{l}\text { Evidence } \\
\text { brief for } \\
\text { policy }\end{array}$ & Maternal and child health & $\begin{array}{l}\text { Delivery } \\
\text { arrangements }\end{array}$ & 2012 & $\begin{array}{l}\text { SURE Project - Makerere } \\
\text { University College of } \\
\text { Health Sciences }\end{array}$ \\
\hline $\begin{array}{l}\text { Evidence } \\
\text { brief for } \\
\text { policy }\end{array}$ & $\begin{array}{l}\text { Governance, coordination } \\
\& \text { M\&E }\end{array}$ & $\begin{array}{l}\text { Governance } \\
\text { arrangements }\end{array}$ & 2012 & $\begin{array}{l}\text { Uganda National Academy } \\
\text { of Sciences }\end{array}$ \\
\hline $\begin{array}{l}\text { Evidence } \\
\text { brief for } \\
\text { policy }\end{array}$ & $\begin{array}{l}\text { Health education, } \\
\text { promotion, environmental } \\
\text { health \& nutrition }\end{array}$ & $\begin{array}{l}\text { Financial } \\
\text { arrangements }\end{array}$ & 2011 & $\begin{array}{l}\text { Uganda National Academy } \\
\text { of Sciences }\end{array}$ \\
\hline $\begin{array}{l}\text { Evidence } \\
\text { brief for } \\
\text { policy }\end{array}$ & $\begin{array}{l}\text { Disease prevention, } \\
\text { mitigation \& control }\end{array}$ & $\begin{array}{l}\text { Delivery } \\
\text { arrangements }\end{array}$ & 2014 & $\begin{array}{l}\text { Uganda National Academy } \\
\text { of Sciences }\end{array}$ \\
\hline $\begin{array}{l}\text { Evidence } \\
\text { brief for } \\
\text { policy }\end{array}$ & $\begin{array}{l}\text { Health education, } \\
\text { promotion, environmental } \\
\text { health \& nutrition }\end{array}$ & $\begin{array}{l}\text { Governance } \\
\text { arrangements }\end{array}$ & 2014 & Uganda Women's Network \\
\hline
\end{tabular}


Table 4 Documents reviewed (Continued)

\begin{tabular}{|c|c|c|c|c|c|c|}
\hline 262 & $\begin{array}{l}\text { What is Needed to Eliminate Mother } \\
\text { to Child Transmission of HIV/AIDS } \\
\text { in Uganda? }\end{array}$ & $\begin{array}{l}\text { Evidence } \\
\text { brief for } \\
\text { policy }\end{array}$ & $\begin{array}{l}\text { Disease prevention, } \\
\text { mitigation \& control }\end{array}$ & $\begin{array}{l}\text { Implementation } \\
\text { strategies }\end{array}$ & 2014 & $\begin{array}{l}\text { Uganda's HIV/AIDS } \\
\text { Knowledge Management } \\
\text { and Communication } \\
\text { Capacity (KMCC) initiative }\end{array}$ \\
\hline 263 & $\begin{array}{l}\text { Expanding Private Health Insurance } \\
\text { Coverage for HIV and AIDS in } \\
\text { Sub-Saharan Africa }\end{array}$ & $\begin{array}{l}\text { Evidence } \\
\text { brief for } \\
\text { policy }\end{array}$ & Health financing & $\begin{array}{l}\text { Financial } \\
\text { arrangements }\end{array}$ & 2013 & USAID \\
\hline 264 & $\begin{array}{l}\text { Tracking Contraceptive Financing } \\
\text { - Lessons from Uganda }\end{array}$ & $\begin{array}{l}\text { Evidence } \\
\text { brief for } \\
\text { policy }\end{array}$ & Health financing & $\begin{array}{l}\text { Financial } \\
\text { arrangements }\end{array}$ & 2013 & USAID \\
\hline 265 & $\begin{array}{l}\text { Accreditation of Institutions for } \\
\text { Health Professional Education }\end{array}$ & $\begin{array}{l}\text { Evidence } \\
\text { brief for } \\
\text { policy }\end{array}$ & $\begin{array}{l}\text { Governance, coordination } \\
\& M \& E\end{array}$ & $\begin{array}{l}\text { Governance } \\
\text { arrangements }\end{array}$ & 2013 & World Health Organization \\
\hline
\end{tabular}

increase in the number of documents from 6 documents per year in 2000 to 49 per year in 2011, dropping in the following years. The lack of documents in the earlier years may not be surprising because, the older they are the harder it is to find such documents online. However, it could also be due to the delay in posting the documents on the website. The increase in the volume of documents over time may reflect an increase in funding for health policy and systems in the country.

The national health priority areas were not equally tackled by the documents, some were more addressed than others. For example, the clusters of disease prevention, mitigation and control and that of governance, coordination, monitoring and evaluation were each covered by

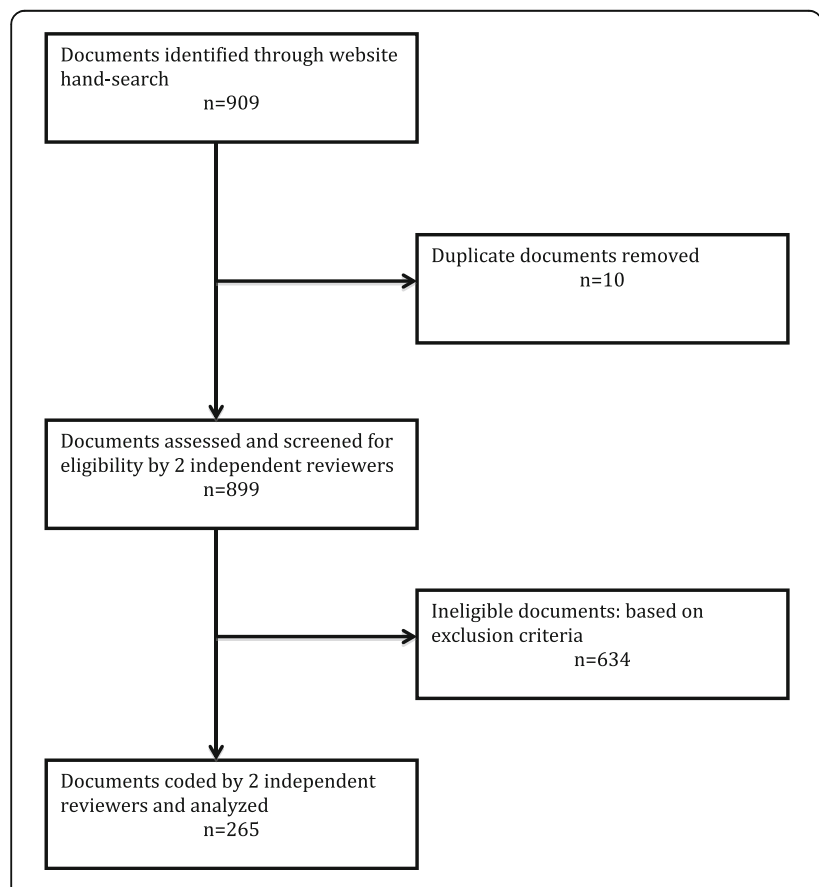

Fig. 2 Results of the document search process and screening almost a quarter of the documents. The breadth of topics and types of documents available reflects what is considered most important by the government of Uganda or may be what the major funding sources perceive to be the most important health issues in Uganda. Besides funding and politics, the sector decision-making process is guided by a sector-wide approach, the compact and International Health Partnerships Plus frameworks involving all key stakeholders, including donors, private sector, civil society and the Government of Uganda. However, these findings may be an indication of the lack of a clear priority-setting mechanism for health policy and systems and of sufficient funding to address important areas.

The delivery arrangement was the most popular health system domain covered by the documents, followed by governance and implementation strategies. A paucity of documents on the financial arrangements domain and the crosscutting issues was noted. This is similar to findings from other studies in low- and middle-income countries [26, 28]. For example, a review by Rao et al. [28] on health systems research in the time of health system reform in India indicated that service delivery was the health system domain most covered by the publications reviewed in comparison to other domains. The neglect of the finance arrangements domains was also noted in Mijumbi's study on the feasibility of a rapid response mechanism to meet policymakers' urgent needs for research evidence about health systems in a lowincome country [26]. The lack of documents in some health system domains, such as financial arrangements, may reflect many reasons such as lack of interest in the area by the authorities.

\section{Strengths and limitations}

To our knowledge, this is the first scoping review of local policy-relevant documents that address questions about health systems and interventions in the eastern African region; previous papers have focused on developed countries in general [14-17]. Our study utilised a 


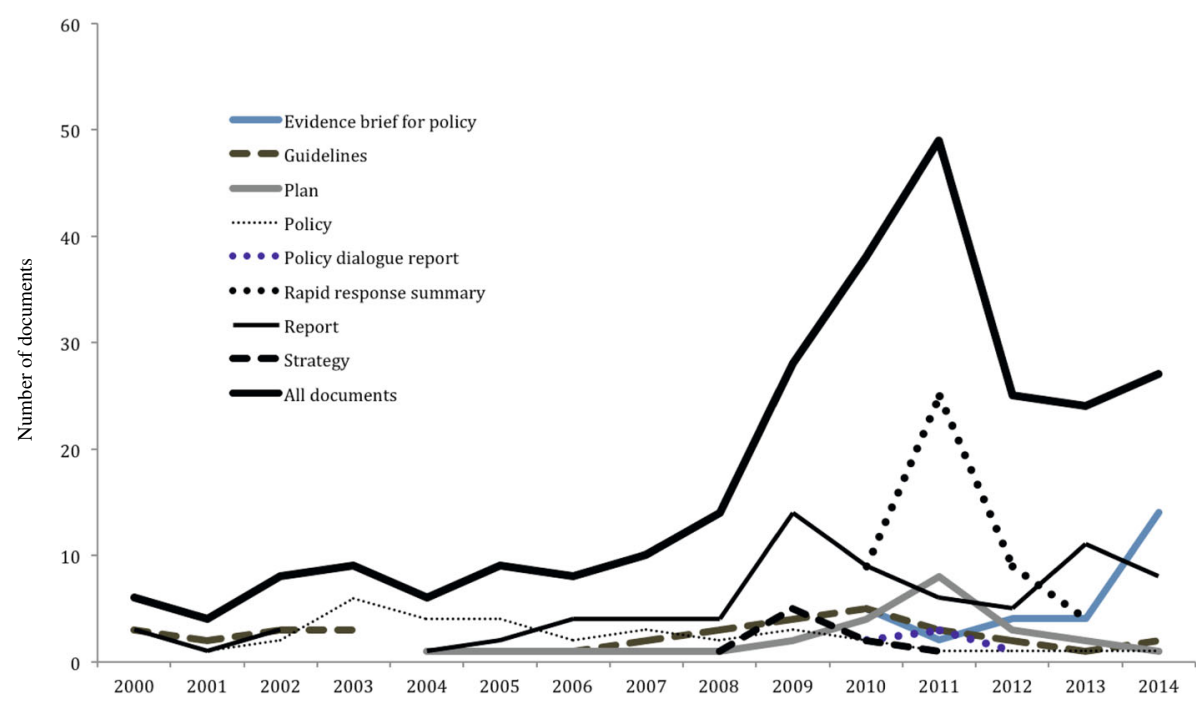

Fig. 3 Number of policy-relevant documents produced by year

rigorous methodological approach for scoping reviews that ensured the validity of the results [21]. We tried to identify all published documents by searching different websites of relevant NGOs and national institutions. The combination of heterogeneous sources of data adds value to the results. However, the review is not exhaustive because we were not able to include hard copies of the documents that were not yet uploaded on the websites. Further, given the wide range of terminology used to describe policy and systems-relevant documents, the study could have missed identifying some documents. We did not consider hard copies due to the limited resource setting. However, we used the website documents as tracers. We recommend future research to consider documents not on websites. Looking at national documents is just one of the inputs in decision-making. International learning can inform local policies. However, our study focused on the Uganda-specific documents as just one of the many inputs.

\section{Implications for policy and research}

Our study provides novel insights into the creation of one-stop shops for research evidence and policy-relevant documents. Specifically, it demonstrates the feasibility of identifying the content of the clearinghouse in a lowand middle-income country, provides an explicit mechanism for categorising the content, and shows that it is possible to adapt the index of health policy documents. Our approach provides academic and other research institutions involved in knowledge brokerage in low- and middle-income countries with a framework for identifying and organising the content of the on-line repositories for health policy and system information. To our knowledge, this is the first scoping review of local policy-relevant documents that address questions about health systems and interventions in the African region. Previous research has focused on developed countries in general.

It is anticipated that this framework may add to the ongoing research efforts in high-income countries that have focused on developing one-stop shops for both global research evidence and local policy-relevant documents. Such efforts include a study by Lavis et al. [15] on health system evidence that focused on developing and refining the methods for a 'one-stop shop' for synthesised research evidence about health systems. In this study, they developed a taxonomy of health system topics for categorising systematic reviews and systematic review protocols. This study demonstrated that policymakers and stakeholders could easily access and use a wide variety of types of research evidence about health systems to inform decision-making and advocacy. Rosenbaum et al. [14] also studied the user experiences of The Cochrane Library, providing a basis for building and improving on-line resources for evidence-based practices. In another study, Faith et al. [17] developed and tested a search tool for HTA Database Canadian Search interface for supporting the use of health technology assessments by decision-makers.

In particular, our findings can inform re-designing of the Uganda Clearinghouse for Health Policy and Systems. The documents reviewed tackle issues identified by the Second Health Policy and National Development Plan, which feed into the Vision 2040 and subsequently contribute to Sustainable Development Goal 3 (i.e. ensure healthy lives and promote well-being for all at all 
ages) $[24,25,29,30]$. Thus, categorising content of the on-line repository according to the national health priority issues may increase the chances of using the resource by health policy and decision-makers. The study findings can also inform government and funders to support the production of policy and systems documents to address the coverage gaps in the national priority issues and health systems domains. In this study, it was not feasible to hold consultations with consumers and stakeholders, future scoping work should consider this for prioritisation, additional sources of information and perspective.

\section{Conclusion}

A one-stop shop for health policy-relevant information may increase the likelihood of using the resource to inform decisions about health systems and interventions if it consists of a wide variety of relevant document types. Thus, the demonstrated availability of health policy and systems documents that address a number of national priority health issues is important for facilitating efforts towards mobilising, building and organising the content of a one-stop shop for Uganda-specific documents. With the resource in place, policymakers, decision-makers and stakeholders will now easily access and use well-packaged policy-relevant documents for decision-making.

\section{Endnotes}

${ }^{1} \mathrm{~A}$ policy is a course or principle of action adopted by the government or executive of a state, intended to guide decisions and achieve rational outcome.

${ }^{2} \mathrm{~A}$ decision is the most logical and suitable course of action about a particular situation selected by the government or executive of a state to achieve the desirable outcome.

${ }^{3} \mathrm{~A}$ policymaker whose action and opinion strongly influences the course of events at an international, national, regional, or local level such as legislators and commissioners at Ministry of Health.

${ }^{4} \mathrm{~A}$ stakeholder is a person (or an interest group) with a powerful bearing on the outcome of policymaking process.

\section{Acknowledgements}

We thank the Research Assistants, the Data Entry Clerks and all who played a role in conducting this study. We also acknowledge the support from SURE project staff, EVIPNet Africa REACH-PI, and Office of the principal - Makerere University College of Health Sciences.

\section{Funding}

The International Development Research Centre (IDRC) - International Research Chair in Evidence-Informed Health Policies and Systems under Makerere University and McMaster University collaboration, funded this study.

Availability of data and materials

The data supporting the results of this study are included in this paper.

\section{Authors' contributions}

MB led the study conceptualisation and design, data analysis, interpretation of findings, writing, and editing. NS and $J L$ guided the conceptualisation and design of the study and contributed to interpretation of findings, writing, and editing of the manuscript. EO and RB contributed to assessing and screening documents for eligibility, coding documents, writing and editing. All authors read and approved the final manuscript.

\section{Competing interests}

The authors declare that they have no competing interests.

\section{Consent for publication}

Not applicable.

Ethics approval and consent to participants

Not applicable.

\section{Author details}

${ }^{1}$ College of Health Sciences, Makerere University, Kampala, Uganda. ${ }^{2}$ International Health Sciences University, Kampala, Uganda. ${ }^{3}$ McMaster Health Forum, Centre for Health Economics and Policy Analysis, Department of Clinical Epidemiology \& Biostatistics, and Department of Political Science, McMaster University, 1280 Main St. West, CRL 209, Hamilton ON L8S 4L6, Ontario, Canada. ${ }^{4}$ Department of Global Health and Population, Harvard T.H. Chan School of Public Health, 677 Huntington Ave., Boston, MA 02115, United States of America. ${ }^{5}$ Faculty of Epidemiology and Population Health, London School of Hygiene and Tropical Medicine, London WC1E 7HT, United Kingdom.

Received: 7 December 2016 Accepted: 16 January 2017

Published online: 06 February 2017

\section{References}

1. Cordero C, Delino R, Jeyaseelan L, Lansang MA, Lozano JM, Kumar S, Moreno S, Pietersen M, Quirino J, Thamlikitkul V, Welch VA, Tetroe J, ter Kuile A, Graham ID, Grimshaw J, Neufeld V, Wells G, Tugwell P. Funding agencies in low- and middle-income countries: support for knowledge translation. Bull World Health Organ. 2008;86(7):524-34.

2. World Health Organization. World report on knowledge for better health: Strengthening health systems. 2004. http://www.who.int/rpc/meetings/en/ world_report_on_knowledge_for_better_health2.pdf. Accessed 15 Jan 2015

3. Lavis JN. Issue Brief: Supporting Quality Improvement in Primary Healthcare in Ontario. 2010. http://www.hqontario.ca/portals/0/Documents/qi/qimcmaster-issue-brief-100621-en.pdf. Accessed 15 Jan 2015.

4. Bucknall T. Bridging the know-do gap in health care through integrated knowledge translation. Worldviews Evid Based Nurs. 2012;9(4):193-4.

5. World Health Organization. Strengthening Health Systems: The role and promise of policy and systems research. 2004. http://www.who.int/alliancehpsr/resources/Strengthening_complet.pdf. Accessed 15 Jan 2015.

6. Oliver K, Lorenc T, Innvær S. New directions in evidence-based policy research: a critical analysis of the literature. Health Res Policy Syst. 2014;12: 34. doi:10.1186/1478-4505-12-34.

7. Greenhalgh T, Russell J. Reframing evidence synthesis as rhetorical action in the policy-making drama. Healthc Policy. 2006;1(2):34-42.

8. Dobrow MJ, Goel V, Lemieux-Charles L, Black NA. The impact of context on evidence utilization: a framework for expert groups developing health policy recommendations. Soc Sci Med. 2006;63(7):1811-24.

9. Santesso N, Tugwell P. Knowledge translation in developing countries. J Contin Educ Health Prof. 2006;26(1):87-96.

10. Innvaer S, Vist G, Trommald M, Oxman A. Health policymakers' perceptions of their use of evidence: a systematic review. J Health Serv Res Policy. 2002; 7:239-44.

11. Lavis J, Davies H, Oxman A, Denis JL, Golden-Biddle K, Ferlie E. Towards systematic reviews that inform health care management and policy-making J Health Serv Res Policy. 2005;10 Suppl 1:35-48.

12. Orton L, Lloyd-Williams F, Taylor-Robinson D, O'Flaherty M, Capewell S. The use of research evidence in public health decision-making processes: systematic review. PLoS One. 2011;6(7):e21704. doi:10.1371/journal.pone.0021704.

13. Oliver K, Innvar S, Lorenc T, Woodman J, Thomas J. A systematic review of barriers to and facilitators of the use of evidence by policymakers. BMC Health Serv Res. 2014;14:2. doi:10.1186/1472-6963-14-2. 
14. Rosenbaum SE, Glenton C, Cracknell J. User experiences of evidence-based online resources for health professionals: user testing of The Cochrane Library. BMC Med Inform Decis Mak. 2008;8:34. doi:10.1186/1472-6947-8-34.

15. Lavis JN, Wilson MG, Moat KA, Hammill AC, Boyko JA, Grimshaw JM, Flottorp S. Developing and refining the methods for a 'one-stop shop' for research evidence about health systems. Health Res Policy Syst. 2015;13:10.

16. Ebenezer C. Usability evaluation of an NHS library website. Health Info Libr J. 2003;20(3):134-42. Erratum in: Health Info Libr J. 2003 Dec; 20(4): 248.

17. Faith A, Bekhuis T. HTA Database Canadian Repository. J Med Libr Assoc 2015;103(4):239-40. doi:10.3163/1536-5050.103.4.021.

18. World Health Organization. http://www.who.int/evidence/sure/en/. Accessed 2 May 2015.

19. World Health Organization. http://global.evipnet.org/about-evipnet/about2/. Accessed 2 May 2015

20. Kirunga TC, Ssengooba F. Health Systems Reforms in Uganda: Processes and Outputs. 2006. http://r4d.dfid.gov.uk/pdf/outputs/healthsysdev_kp/uganda_ book.pdf. Accessed 10 March 2015.

21. Arksey H, O'Malley L. Scoping studies: towards a methodological framework. Int J Soc Res Methodol. 2005;8(1):19-32.

22. Robb M, Shellenbarger T. Strategies for searching and managing evidence-based practice resources. J Contin Educ Nurs. 2014;45(10):461-6.

23. Hoffman S. Conceptual Issues Related to Health Systems Research to Inform a WHO Global Strategy on Health Systems Research. 2012. http://www.who.int/ alliance-hpsr/alliancehpsr_backgroundpaperhsrstrat1.pdf. Accessed 4 Dec 2014.

24. National Planning Authority. Second National Development Plan - NDPII 2015/16 - 2019/20. 2015. http://npa.ug/wp-content/uploads/NDPII-Final.pdf. Accessed 2 June 2015

25. Ministry of Health. Second National Health Policy. 2010. http://library.health. go.ug/publications/leadership-and-governance-governance/policydocuments/second-national-health-policy. Accessed 2 June 2015.

26. Mijumbi RM, Oxman AD, Panisset U, Sewankambo NK. Feasibility of a rapid response mechanism to meet policymakers' urgent needs for research evidence about health systems in a low-income country: a case study Implement Sci. 2014;9:114.

27. Murphy GT, Goma F, MacKenzie A, Bradish S, Price S, Nzala S, Rose AE, Rigby J, Muzongwe C, Chizuni N, Carey A, Hamavhwa D. A scoping review of training and deployment policies for human resources for health for maternal, newborn, and child health in rural Africa. Hum Resour Health. 2014;12:72.

28. Rao KD, Arora R, Ghaffar A. Health systems research in the time of health system reform in India: a review. Health Res Policy Syst. 2014;12:37. doi:10. 1186/1478-4505-12-37.

29. United Nations. Transforming our world: the 2030 Agenda for Sustainable Development: United Nations Department of Economic and Social Affairs. 2015. http://www.un.org/ga/search/view_doc.asp?symbol=A/RES/70/ 1\&Lang=E. Accessed 4 Jan 2015.

30. Uganda National Planning Authority. Uganda Vision 2040. 2015. http://npa.ug/uganda-vision-2040. Accessed 4 Jan 2015.

\section{Submit your next manuscript to BioMed Central and we will help you at every step:}

- We accept pre-submission inquiries

- Our selector tool helps you to find the most relevant journal

- We provide round the clock customer support

- Convenient online submission

- Thorough peer review

- Inclusion in PubMed and all major indexing services

- Maximum visibility for your research

Submit your manuscript at www.biomedcentral.com/submit

CBiomed Central 